\title{
Charged jet cross section and fragmentation in proton-proton collisions at $\sqrt{s}=7 \mathrm{TeV}$
}

\author{
S. Acharya et al. \\ (A Large Ion Collider Experiment Collaboration)
}

(Received 14 September 2018; published 31 January 2019)

\begin{abstract}
We report the differential charged jet cross section and jet fragmentation distributions measured with the ALICE detector in proton-proton collisions at a center-of-mass energy $\sqrt{s}=7 \mathrm{TeV}$. Jets with pseudorapidity $|\eta|<0.5$ are reconstructed from charged particles using the anti- $k_{\mathrm{T}}$ jet-finding algorithm with a resolution parameter $R=0.4$. The jet cross section is measured in the transverse momentum interval $5 \leq p_{\mathrm{T}}^{\text {ch jet }}<100 \mathrm{GeV} / c$. Jet fragmentation is studied measuring the scaled transverse momentum spectra of the charged constituents of jets in four intervals of jet transverse momentum between 5 and $30 \mathrm{GeV} / c$. The measurements are compared to calculations from the PYTHIA model as well as next-to-leading-order perturbative QCD calculations with POWHEG+PYTHIA8. The charged jet cross section is well described by POWHEG for the entire measured range of $p_{\mathrm{T}}^{\text {ch jet }}$. For $p_{\mathrm{T}}^{\text {ch jet }}>40 \mathrm{GeV} / c$, the PYTHIA calculations also agree with the measured charged jet cross section. PYTHIA6 simulations describe the fragmentation distributions to $15 \%$. Larger discrepancies are observed for PYTHIA8.
\end{abstract}

DOI: 10.1103/PhysRevD.99.012016

\section{INTRODUCTION}

The measurement of jets in proton-proton (pp) collisions allows the study of hard scatterings and subsequent fragmentation of partons (quarks and gluons). In this work, measurements of the charged jet cross section and jet fragmentation in $\mathrm{pp}$ collisions at $\sqrt{s}=7 \mathrm{TeV}$ are presented. The inclusive charged jet cross section is measured in the transverse momentum $\left(p_{\mathrm{T}}\right)$ range $5 \leq$ $p_{\mathrm{T}}^{\text {ch jet }}<100 \mathrm{GeV} / c$. For sufficiently high $p_{\mathrm{T}}$, jet production cross sections can be calculated in perturbative quantum chromodynamics (pQCD) supplemented with parton distribution functions (PDFs), assuming collinear factorization. Soft processes (e.g., production of particles or prompt photons with $p_{\mathrm{T}} \lesssim 2 \mathrm{GeV} / c$ [1-3]) cannot be described by this formalism. The measurements presented in this paper test the applicability of $\mathrm{pQCD}$ on jet production [4] down to a kinematic regime of the order of a few $\mathrm{GeV} / c$ and provide experimental constraints on the PDFs (see e.g., Ref. [5]). Quantitative pQCD predictions for the charged jet cross sections are obtained in the POWHEG [6-8] scheme, in which matrix elements are calculated at next-to-leading order (NLO) in the QCD

*Full author list given at the end of the article.

Published by the American Physical Society under the terms of the Creative Commons Attribution 4.0 International license. Further distribution of this work must maintain attribution to the author(s) and the published article's title, journal citation, and DOI. coupling and matched to parton shower Monte Carlo (MC) event generators to simulate parton fragmentation.

In Ref. [2], it was found that NLO pQCD overestimates the measured cross sections for inclusive $\pi^{0}$ and $\eta$ meson production at $\sqrt{s}=7 \mathrm{TeV}$. Perturbative QCD calculations of hadron production rely strongly on parton to hadron fragmentation functions [9], whereas for jet observables this dependence is much smaller. The measured charged jet cross sections help to trace the origin of this observed discrepancy.

The production cross sections of jets in pp collisions at $\sqrt{s}=7 \mathrm{TeV}$ were measured previously by the ATLAS Collaboration for $100 \leq p_{\mathrm{T}}^{\text {jet }}<2000 \mathrm{GeV} / c$ [10] and in the charged jet $p_{\mathrm{T}}$ range $4 \leq p_{\mathrm{T}}^{\text {ch jet }}<100 \mathrm{GeV} / c[11]$ and by the CMS Collaboration for $18 \leq p_{\mathrm{T}}^{\text {jet }}<1100 \mathrm{GeV} / c$ [12] and $100 \leq p_{\mathrm{T}}^{\text {jet }}<2000 \mathrm{GeV} / c$ [13]. Jet fragmentation in $\mathrm{pp}$ and $\mathrm{Pb}-\mathrm{Pb}$ collisions at the LHC were reported by ATLAS [11,14,15] and CMS [16]. In Ref. [17], the ALICE Collaboration measured charged jet cross sections and leading jet properties for $20 \leq p_{\mathrm{T}}^{\text {chjet leading }}<100 \mathrm{GeV} / c$. An approximate scaling of the fragmentation distributions with the fractional transverse momentum $z^{\text {ch }}=p_{\mathrm{T}}^{\text {particle }} / p_{\mathrm{T}}^{\text {chjet }}$ was observed for $z^{\mathrm{ch}}>0.1$ and the distributions were found to be similar for the reported $p_{\mathrm{T}}^{\text {ch jet leading }}$ range. The results presented in this work repeat the previous measurements for a jet resolution parameter of anti- $k_{\mathrm{T}}$ [18] jets with $R=0.4$ with smaller uncertainties and an extended jet $p_{\mathrm{T}}$ coverage. The distributions of the fractional transverse momentum $z^{\text {ch }}$ 
of particles in jets with $5 \leq p_{\mathrm{T}}^{\text {ch jet }}<30 \mathrm{GeV} / c$ presented in this work test the fragmentation scaling for lower jet $p_{\mathrm{T}}$. Furthermore, they provide constraints on the parton shower and hadronization models in MC event generators in a kinematic regime where strong nonperturbative effects are expected. In commonly used event generators, soft particle production is modeled by hard parton fragmentation and multiparton interactions, evoking nonperturbative color reconnection $[19,20]$ at hadronization. The present results allow the perturbative contribution to inclusive particle production to be quantified and also allow for tests of color reconnection effects on the fragmentation of jets with $p_{\mathrm{T}}^{\text {ch jet }}>5 \mathrm{GeV} / c$.

This paper is organized as follows. Section II describes the experiment and detectors used for these measurements. The observables and the details of the jet reconstruction algorithms and parameters are discussed in Sec. III. Section IV discusses the MC simulations carried out for comparisons of data to models, corrections for instrumental effects, and systematic uncertainty studies. The procedures applied to correct for instrumental effects are described in Sec. V. The methods used to evaluate the systematic uncertainties of the measurements are discussed in Sec. VI. Results are presented and discussed in comparison with MC event generator simulations in Sec. VII. Section VIII summarizes the results and conclusions.

\section{EXPERIMENTAL SETUP AND DATA SAMPLE}

The data used in this analysis were collected during the 2010 LHC pp run with the ALICE detector [21]. The analysis relies primarily on the time projection chamber (TPC) [22], the inner tracking system (ITS) [23], and the V0 [24] subdetectors. The V0 and ITS are used for event selection. The results reported in this paper are based on $177 \times 10^{6}$ minimum bias events corresponding to an integrated luminosity of $(2.9 \pm 0.1) \mathrm{nb}^{-1}$ [25]. The minimum bias trigger requires at least one hit in either the V0 forward scintillators or in the two innermost silicon pixel detector layers (SPD) of the ITS, in coincidence with a LHC bunch crossing. The TPC and ITS are used for primary vertex and track reconstruction. Only events with a primary vertex within $\pm 10 \mathrm{~cm}$ along the beam direction from the nominal interaction point are analyzed to minimize dependencies of the TPC acceptance on the vertex position.

Charged tracks are reconstructed using the combined information from the TPC and the ITS within $|\eta|<0.9$ over the full azimuth $(\varphi)$. The track selection criteria are the same as described in Ref. [17] and are briefly outlined here. To assure a uniform $\varphi$ distribution, a hybrid reconstruction technique is utilized, combining two distinct track classes: (i) tracks containing from three to six hits in the ITS, including at least one hit in the SPD, and (ii) tracks containing fewer than three hits in the ITS, or no hit in the SPD. The momentum of tracks of class (i) is determined without a vertex constraint. The vertex constraint is added for class (ii) tracks to improve the determination of their transverse momentum. The track momentum resolution $\delta p_{\mathrm{T}} / p_{\mathrm{T}}$ is approximately $4 \%$ at $p_{\mathrm{T}}=40 \mathrm{GeV} / c$ for $95 \%$ of all tracks. For tracks without a hit in the ITS $(5 \%$ of the track sample) the resolution is $7 \%$ at $p_{\mathrm{T}}=40 \mathrm{GeV} / c$. Tracks from primary particles are selected requiring a minimum distance of closest approach to the primary vertex of $2.4 \mathrm{~cm}$ in the plane transverse to the beam and $3.2 \mathrm{~cm}$ in the beam direction.

To ensure good momentum resolution, tracks in the TPC are selected requiring a $p_{\mathrm{T}}$-dependent minimum number of space points and a maximum $\chi^{2}$ to ensure track fit quality. In addition, there is an upper threshold on the $\chi^{2}$ between the results of the track fit using all the space points in the ITS and TPC, and using only the TPC space points with the primary vertex position as an additional constraint.

The track reconstruction efficiency for primary charged particles is approximately $60 \%$ at $p_{\mathrm{T}}=0.15 \mathrm{GeV} / c$, about $87 \%$ at $1 \mathrm{GeV} / c$, and is nearly uniform up to $10 \mathrm{GeV} / c$ beyond which it decreases slightly. The efficiency is roughly uniform in azimuth and within the pseudorapidity range $|\eta|<0.9$. Further details on the track selection procedure and tracking performance can be found in Refs. $[17,26]$.

\section{JET RECONSTRUCTION AND OBSERVABLES}

The anti- $k_{\mathrm{T}}$ [18] algorithm from the FASTJET package [27] is used for charged jet reconstruction. Jets with a resolution parameter $R=0.4$ are reconstructed from charged tracks with $p_{\mathrm{T}}>0.15 \mathrm{GeV} / c$ and within $|\eta|<0.9$. The analyses reported in this work are restricted to jets detected within the fiducial acceptance $|\eta|<0.5$. A boost-invariant $p_{\mathrm{T}}$ recombination scheme is used to determine the transverse momenta of jets as the sum of their charged particle transverse momenta.

The cross section is evaluated with

$$
\frac{\mathrm{d}^{2} \sigma^{\text {ch jet }}}{\mathrm{d} p_{\mathrm{T}} \mathrm{d} \eta}\left(p_{\mathrm{T}}^{\text {chjet }}\right)=\frac{1}{\mathcal{L}^{\text {int }}} \frac{\Delta N_{\text {jets }}}{\Delta p_{\mathrm{T}} \Delta \eta}\left(p_{\mathrm{T}}^{\text {ch jet }}\right),
$$

where $\mathcal{L}^{\text {int }}$ is the integrated luminosity and $\Delta N_{\text {jets }}$ is the number of jets in the selected intervals of $\Delta p_{\mathrm{T}}$ and $\Delta \eta$.

The jet fragmentation is reported based on the distribution

$$
F^{z}\left(z^{\mathrm{ch}}, p_{\mathrm{T}}^{\text {ch jet }}\right)=\frac{1}{N_{\text {jets }}} \frac{\mathrm{d} N}{\mathrm{~d} z^{\text {ch }}},
$$

where $N$ is the number of charged particles. The scaled $p_{\mathrm{T}}$ variable $z^{\text {ch }}$ is calculated jet by jet for each track. 
This observable characterizes the longitudinal jet fragmentation parallel to the jet axis.

\section{MONTE CARLO SIMULATIONS}

Simulations of the ALICE detector performance for particle detection and jet reconstruction are used to correct the measured distributions for instrumental effects, evaluate systematic uncertainties, and make model comparisons to data. The simulated and real data are analyzed with the same reconstruction algorithms. The nominal corrections are calculated from simulated events generated with the PYTHIA 6.425 [28] (tune Perugia-0 [29]) MC model and particles are transported with GEANT3 [30]. The MC models HERWIG 6.510 [31,32] and several PYTHIA6 tunes are used for systematic investigations of the sensitivity of the MC correction factors to variations of the detector response (described in Sec. V) as well as to jet fragmentation and hadronization patterns (as described in Secs. VIA and VIB). For comparison to data in Sec. VII, PYTHIA6, PYTHIA8 [33], and POWHEG+PYTHIA8 simulations are used.

PYTHIA and HERWIG are leading-order (LO) event generators based on pQCD calculations of $(2 \rightarrow 2)$ hard scattering elements. Higher-order emissions are included in the parton shower. PYTHIA and HERWIG utilize different approaches to describe the parton shower and hadronization processes. HERWIG makes angular ordering a direct part of the evolution process and thereby takes coherence effects into account in the emission of soft gluons. PYTHIA6.4 is based on transverse-momentum-ordered showers [34] in which angular ordering is imposed by an additional veto. In PYTHIA6 the initial-state evolution and multiple parton-parton interactions are interleaved into one common decreasing $p_{\mathrm{T}}$ sequence. In PYTHIA8 the final-state evolution is also interleaved with initial-state radiation and multiparton interactions. Hadronization in PYTHIA proceeds via string breaking as described by the Lund model [35], whereas HERWIG uses cluster fragmentation.

The PYTHIA Perugia tune variations, beginning with the central tune Perugia-0 [29], are based on LEP, Tevatron, and SPS data. The PYTHIA6 Perugia-2011 family of tunes [29] belongs to the first generation of tunes that use LHC pp data at $\sqrt{s}=0.9$ and $7 \mathrm{TeV}$. For the PYTHIA8 Monash tune [36] data at $\sqrt{s}=8$ and $13 \mathrm{TeV}$ are also used. The HERWIG generator version and PYTHIA tunes used in this work utilize the CTEQ5L parton distributions [5]. The PYTHIA8.21 Monash tune uses the NNPDF2.3 LO set [37].

The POWHEG BOX framework [7,8], an event-by-event $\mathrm{MC}$, was used for $\mathrm{pQCD}$ calculations of $(2 \rightarrow 2)$ and $(2 \rightarrow 3)$ parton scattering at $\mathcal{O}\left(\alpha_{S}^{3}\right)$ in the strong coupling constant. The outgoing partons from POWHEG are passed to PYTHIA8 event by event where the subsequent parton shower is handled. The MC approach has the advantage that the same selection criteria and jet finding algorithm can be used on the final-state particle level as used in the analysis of the real data; in particular, charged particles can be selected. For the comparison with the measured differential jet cross sections, the CTEQ6M parton distribution functions [38] are used [39]. The dominant uncertainty in the parton-level calculation is given by the choice of renormalization scale, $\mu_{\mathrm{R}}$, and factorization scale, $\mu_{\mathrm{F}}$. The default value was chosen to be $\mu_{\mathrm{R}}=\mu_{\mathrm{F}}=p_{\mathrm{T}}$ of the underlying Born configuration, here a QCD $2 \rightarrow 2$ scattering [8]. Independent variations by a factor of 2 around the central value are considered as the systematic uncertainty. In addition, the uncertainty on the parton distribution functions has been taken into account by the variation of the final results for the respective error sets of the PDFs. For the POWHEG calculations, PYTHIA8 tune Monash was used. For test purposes, the calculations were repeated with multiparton interactions (MPI) switched off as an alternative setting.

\section{CORRECTIONS}

The measured jet spectra and fragmentation distributions are corrected to the primary charged particle level, as discussed in the following sections.

\section{A. Unfolding}

Momentum-dependent imperfections in the particle detection efficiency and the finite track momentum resolution of the detector affect the jet energy scale and jet fragmentation distributions reported in this work. A detector response matrix is used to correct the jet spectra and fragmentation distributions for these effects. The instrumental response is modeled in a full simulation of the ALICE detector. Simulated events are generated with PYTHIA and the produced particles are transported with GEANT3. Jets are reconstructed both directly from the charged particle momenta produced by the MC generators (particle level) and from the generator outputs processed through GEANT and the ALICE reconstruction software (detector level).

The jet production cross sections and fragmentation distributions are corrected by one- and two-dimensional Bayesian unfolding [40], respectively, as implemented in the RooUnfold [41] software. For the unfolding of the jet cross sections, a two-dimensional response matrix of particle-level versus detector-level charged jet $p_{\mathrm{T}}$ is used. The entries of the response matrix are computed pairing particle- and detector-level jets geometrically, according to the distance $d=\sqrt{\Delta \eta^{2}+\Delta \phi^{2}}$ between the jet axes. A bijective match with $d<0.3$ is required. At the smallest jet $p_{\mathrm{T}}$ presented in this work, $p_{\mathrm{T}}^{\text {ch jet }}=5 \mathrm{GeV} / c$, the combined efficiency of jet reconstruction and matching detector- and particle-level jets exceeds $95 \%$, and rises as a function of $p_{\mathrm{T}}^{\text {chjet }}$ to reach a value $>99 \%$ at $p_{\mathrm{T}}^{\text {chjet }}=20 \mathrm{GeV} / c$. The fragmentation distributions are corrected with a four-dimensional response matrix with the axes corresponding to particle- and detector-level charged jet $p_{\mathrm{T}}$ 
and particle- and detector-level $z^{\text {ch }}$, respectively. Particlelevel and detector-level jet constituents used in the calculation of $z^{\text {ch }}$ are associated by matching the simulated TPC clusters on tracks to hits along the particle trajectories.

In the Bayesian approach, the unfolding solution is regularized by the choice of the number of iterations. We observe that the unfolded distributions typically converge to a solution after five steps. To avoid biases for the lowest and highest values of jet $p_{\mathrm{T}}$ reported in this paper, a wide range $0<p_{\mathrm{T}}^{\text {chjet }}<200 \mathrm{GeV} / c$ is chosen for the uncorrected distributions. Consistency of the unfolding procedure is ensured by folding the solution to the detector level and comparing it to the uncorrected distribution used as input. As an additional cross-check, the analysis of charged jet cross sections is also carried out with the RoOUNFOLD implementation of the singular value decomposition unfolding technique $[41,42]$. Consistent results are obtained with both methods.

The requirement of a match between the simulated detector- and particle-level jets used to compute the response matrix introduces a kinematic bias towards higher momentum constituents. The effect is largest for the fragmentation distribution observable, where it is of the order of $5 \%$ for small values of $z^{\text {ch }}$ and $5 \leq p_{\mathrm{T}}^{\text {chjet }}<$ $10 \mathrm{GeV} / c$. The bias decreases for higher values of $z^{\text {ch }}$ and $p_{\mathrm{T}}^{\text {ch jet }}$. For the jet cross section observable it is less than $0.5 \%$. We account for this effect by applying a correction to the measured distributions prior to unfolding. The correction and the unfolding procedure are validated by $\mathrm{MC}$ closure checks, which will be discussed in Sec. VI.

\section{B. Contamination from secondary particles}

Secondary charged particles are produced by weak decays of strange particles (e.g., $K_{\mathrm{S}}^{0}$ and $\Lambda$ ), decays of charged pions, conversions of photons from neutral pion decays and hadronic interactions in the detector material. Although the contribution of secondaries is minimized by the track selection described in Sec. II, the measured distributions nonetheless must be corrected for a small residual contamination.

The correction for secondary particle contamination is implicitly included in the unfolding of the measured cross sections. It is however carried out separately and explicitly prior to unfolding in the measurements of the fragmentation function, following the procedure described in Ref. [17]. The contribution of secondaries is estimated from MC simulations, separately for each bin in jet $p_{\mathrm{T}}^{\text {ch jet }}$ and particle $z^{\text {ch }}$. The explicit subtraction allows for the enhancement of the low strangeness yield in the PYTHIA Perugia-0 simulations to the level observed in data. Strange particle production in non-single-diffractive events by the CMS Collaboration [43] and MC simulations from Refs. [44,45] are compared. The MC predictions are scaled up to match the data. The contamination of secondaries from strange particle decays is small, and the effect of the strangeness scaling on the final result is less than $1 \%$.

\section{Underlying event subtraction}

The underlying event (UE) corresponds to all particles in an event that are not produced directly by the hard scattering of partons. UE particles emitted in the jet cone contribute to the reconstructed jet $p_{\mathrm{T}}$. To estimate and subtract the UE activity, we use the approach discussed in Ref. [17]. The UE particle yield is measured event by event based on circular regions transverse to the axis of the leading (highest $p_{\mathrm{T}}$ ) jet. The circular regions have the same radius as the jet resolution parameter and are placed at the same pseudorapidity as the leading jet but offset at an azimuthal angle $\Delta \varphi=\pi / 2$ relative to the jet axis. For the jet cross section measurements, the UE is subtracted on a jet-by-jet basis prior to unfolding. The relative UE contribution to the total measured jet $p_{\mathrm{T}}$ is largest for the soft jets. The correction results in a reduction of the uncorrected jet yield by approximately $25 \%$ for $p_{\mathrm{T}}^{\text {ch jet }}=5 \mathrm{GeV} / c$ and by about $10 \%$ for $p_{\mathrm{T}}^{\text {ch jet }}=20 \mathrm{GeV} / c$.

The method used in Ref. [17] to correct the fragmentation distributions in jets with $p_{\mathrm{T}}^{\text {ch jet leading }} \geq 20 \mathrm{GeV} / c$ for the UE applies a subtraction on the level of the constituent spectra, but does not include a simultaneous correction to $p_{\mathrm{T}}^{\text {ch jet }}$. For low- $p_{\mathrm{T}}$ jets, this approximation may not be valid. Therefore, in this work the fragmentation distributions are presented without correction for the UE.

\section{SYSTEMATIC UNCERTAINTIES}

A summary of all systematic uncertainties for the cross section and fragmentation measurements is given in Table I for selected bins in $p_{\mathrm{T}}^{\text {chjet }}$ and $z^{\text {ch }}$ to illustrate the range of systematic uncertainties.

\section{A. Tracking efficiency and resolution}

Uncertainties associated with the momentum resolution and charged track reconstruction efficiency lead to systematic uncertainties in measurements of the jet cross section and jet fragmentation distributions. The relative systematic uncertainty on tracking efficiency is estimated to be $4 \%$ based on variations of track selection criteria. The track momentum resolution has a relative systematic uncertainty of $20 \%$ [46].

The impact of the finite detector efficiency and momentum resolution on the unfolded jet cross sections and fragmentation distributions is estimated by applying a parametrized detector response to PYTHIA events clustered with FASTJET. The efficiency and resolution are varied independently, and a response matrix is computed for each variation. The measured distributions are unfolded, and the resulting variations are used to estimate the systematic uncertainties. The systematic uncertainty on the jet cross 
TABLE I. Summary of systematic uncertainties of the cross section and fragmentation distributions for selected bins in $p_{\mathrm{T}}^{\text {ch jet }}$ and $z^{\text {ch }}$. The contributions from tracking efficiency and track $p_{\mathrm{T}}$ resolution, the event generator dependence of the unfolding correction, $\mathrm{MC}$ closure, secondaries correction, UE subtraction and cross section normalization as well as the total uncertainty are shown.

\begin{tabular}{|c|c|c|c|c|c|c|c|c|c|}
\hline Distribution & Bin & $\begin{array}{c}\text { Track } \\
\text { eff. }(\%)\end{array}$ & $\begin{array}{c}\text { Track } \\
p_{\mathrm{T}} \text { res. }(\%)\end{array}$ & $\begin{array}{c}\text { Event } \\
\text { Generator }(\%)\end{array}$ & $\begin{array}{c}\text { MC } \\
\text { Closure }(\%)\end{array}$ & $\begin{array}{c}\text { Sec. } \\
\text { corr. }(\%)\end{array}$ & $\begin{array}{l}\mathrm{UE} \\
(\%)\end{array}$ & $\begin{array}{c}\text { Norm. } \\
(\%)\end{array}$ & $\begin{array}{c}\text { Total } \\
(\%)\end{array}$ \\
\hline$\frac{\mathrm{d}^{2} \sigma^{\text {ch jet }}}{\mathrm{d} p_{\mathrm{T}}^{\text {ch jet }}}$ & $\begin{array}{c}5-6 \mathrm{GeV} / c \\
20-24 \mathrm{GeV} / c \\
86-100 \mathrm{GeV} / c\end{array}$ & $\begin{array}{r}7.0 \\
10.2 \\
11.7\end{array}$ & $\begin{array}{l}0.1 \\
0.5 \\
2.0\end{array}$ & $\begin{array}{l}2.1 \\
1.0 \\
1.0\end{array}$ & $\begin{array}{l}\cdots \\
\cdots \\
\cdots\end{array}$ & $\begin{array}{l}2.0 \\
2.2 \\
2.6\end{array}$ & $\begin{array}{l}1.7 \\
0.5 \\
1.5\end{array}$ & $\begin{array}{l}3.5 \\
3.5 \\
3.5\end{array}$ & $\begin{array}{r}8.6 \\
11.1 \\
12.7\end{array}$ \\
\hline $\begin{array}{l}\frac{1}{N_{\text {jets }}} \frac{\mathrm{d} N}{\mathrm{~d} z^{\mathrm{ch}}} 5 \leq p_{T}^{\text {ch jet }} \\
\quad<10 \mathrm{GeV} / c\end{array}$ & $\begin{array}{c}0-0.1 \\
0.35-0.4 \\
0.95-1.0\end{array}$ & $\begin{array}{r}4.1 \\
0.1 \\
10.4\end{array}$ & $\begin{array}{c}\text { Negligible } \\
0.2 \\
0.6\end{array}$ & $\begin{array}{l}1.4 \\
2.9 \\
4.7\end{array}$ & $\begin{array}{l}1.0 \\
1.0 \\
1.0\end{array}$ & $\begin{array}{l}3.2 \\
0.6 \\
0.2\end{array}$ & $\begin{array}{l}\cdots \\
\cdots \\
\cdots\end{array}$ & $\begin{array}{l}\cdots \\
\cdots \\
\cdots\end{array}$ & $\begin{array}{r}5.5 \\
3.2 \\
11.4\end{array}$ \\
\hline $\begin{array}{l}\frac{1}{N_{\text {jets }}} \frac{\mathrm{d} N}{\mathrm{~d} z^{\text {ch }}} 15 \leq p_{T}^{\text {ch jet }} \\
\quad<20 \mathrm{GeV} / c\end{array}$ & $\begin{array}{c}0-0.1 \\
0.35-0.4 \\
0.95-1.0\end{array}$ & $\begin{array}{l}4.0 \\
0.8 \\
9.0\end{array}$ & $\begin{array}{c}\text { Negligible } \\
0.7 \\
1.9\end{array}$ & $\begin{array}{l}0.6 \\
0.9 \\
2.5\end{array}$ & $\begin{array}{l}1.0 \\
1.0 \\
1.0\end{array}$ & $\begin{array}{l}2.6 \\
0.4 \\
0.5\end{array}$ & $\begin{array}{l}\cdots \\
\cdots \\
\cdots\end{array}$ & $\begin{array}{l}\cdots \\
\cdots \\
\cdots\end{array}$ & $\begin{array}{l}4.9 \\
1.7 \\
9.6\end{array}$ \\
\hline
\end{tabular}

sections related to tracking efficiency increases smoothly with increasing $p_{\mathrm{T}}^{\text {ch jet }}$. For the fragmentation distributions, the uncertainty is largest at $z^{\text {ch }}=1$ and has a minimum at $z^{\text {ch }} \approx 0.35$. The systematic uncertainty on the measured cross sections and fragmentation distributions from finite momentum resolution is comparatively small, and largest for high $p_{\mathrm{T}}^{\text {ch jet }}$ and $z^{\text {ch }}$.

\section{B. Unfolding}

The data correction methods used in this work are largely based on tune Perugia- 0 of the PYTHIA event generator. The particular structure of jets simulated by PYTHIA might however affect the simulated detector response and influence the correlation between particle- and detectorlevel quantities used to compute the response matrices. Furthermore, the RooUnFold Bayesian unfolding algorithm is based on a prior solution which is initially obtained from the MC and updated in subsequent iterations. The choice of a particular initial prior might have an impact on the unfolded solution. Such event generator dependencies are examined by comparing unfolded solutions obtained with response matrices from the PYTHIA tunes Perugia-0 and Perugia-2011 with those obtained with the HERWIG generator. This is accomplished with a parametrized detector response and the anti- $k_{\mathrm{T}}$ jet finder. The resulting systematic uncertainties on the jet cross sections are largest for the lowest $p_{\mathrm{T}}^{\text {chjet }}$. For the fragmentation distributions, the strongest event generator dependence is observed for the lowest jet $p_{\mathrm{T}}$, in the interval $5 \leq p_{\mathrm{T}}^{\text {ch jet }}<10 \mathrm{GeV} / c$, where the uncertainty is largest for intermediate values of $z^{\text {ch }} \approx 0.4$ and for $z^{\text {ch }}=1$. The distributions for $p_{\mathrm{T}}^{\text {ch jet }} \geq$ $10 \mathrm{GeV} / c$ show a monotonic increase of the systematic uncertainty with $z^{\text {ch }}$.

The unfolding approach is validated by closure tests on PYTHIA simulations. To detect potential biases, the simulated detector-level distribution is unfolded and the solution is compared to the particle-level truth. For the unfolded jet cross section, no significant difference is observed. For the fragmentation distributions, a small systematic bias can be detected. We assign a constant uncertainty of $1 \%$ to account for this nonclosure.

\section{Correction for secondary charged particles}

The systematic uncertainty associated to the correction for the contribution from secondary charged particles to the jet cross sections and fragmentation distributions is estimated by varying track selection criteria. We change the contribution of secondary charged particles by varying the track selection criteria [17] and correct the measured distributions accordingly. Residual variations of the corrected distributions are used to estimate the systematic uncertainties. The resulting uncertainties on the fragmentation distributions are largest at small values of $z^{\text {ch }}$. The uncertainty on the measured jet cross section is evaluated as a $p_{\mathrm{T}}^{\text {chjet }}$ scale uncertainty of $0.5 \%$.

\section{Underlying event subtraction}

The jet cross sections are corrected for the contribution from the UE. In Ref. [17], the uncertainty on the measurement of the UE $p_{\mathrm{T}}$ density was estimated to be $5 \%$. The corresponding uncertainty of the jet cross section is evaluated as a jet $p_{\mathrm{T}}$ scale uncertainty resulting in a systematic uncertainty which is $2 \%$ for $p_{\mathrm{T}}^{\text {chjet }}=5 \mathrm{GeV} / c$ and decreases for higher $p_{\mathrm{T}}^{\text {ch jet }}$.

\section{RESULTS}

Figure 1 presents the inclusive charged jet cross section measured in pp collisions at $\sqrt{s}=7 \mathrm{TeV}$ using the anti- $k_{\mathrm{T}}$ jet finder. The cross section is reported for a resolution parameter $R=0.4$ in the pseudorapidity interval $|\eta|<0.5$. Statistical uncertainties are displayed as vertical error bars. The total systematic uncertainties are obtained as a quadratic sum of the individual contributions described in 


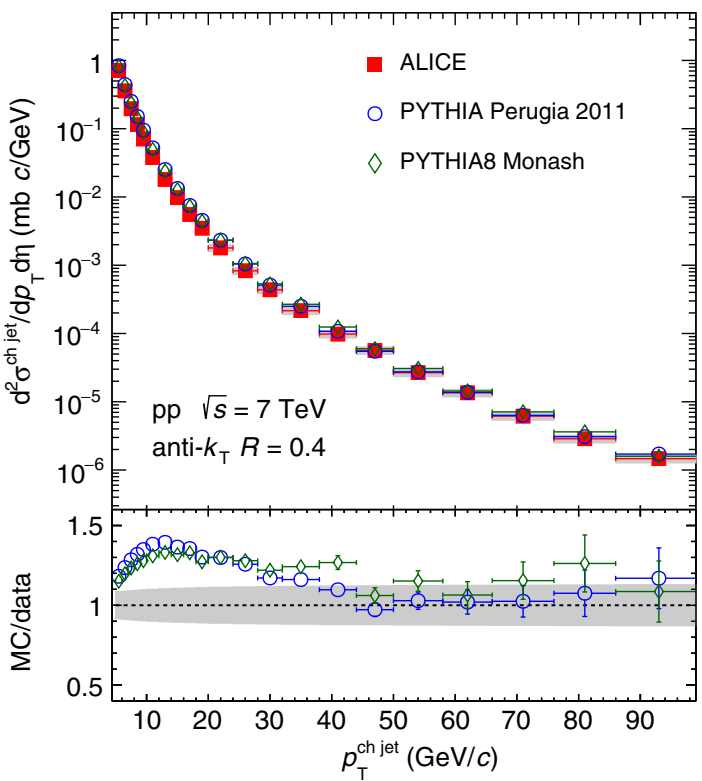

FIG. 1. Top panel: Inclusive charged jet cross section in pp collisions at $\sqrt{s}=7 \mathrm{TeV}$ using the anti- $k_{\mathrm{T}}$ algorithm with $R=$ 0.4 compared to calculations from PYTHIA6 Perugia-2011 and PYTHIA8 tune Monash. Bottom panel: Ratios of MC distributions to data. The shaded band shows the systematic uncertainty on the data drawn at unity, error bars represent the statistical uncertainties. Most uncertainties are smaller than the marker size.

Sec. VI, and are shown as shaded boxes around the data points. The results presented in this work extend the jet $p_{\mathrm{T}}^{\text {ch jet }}$ coverage of previous measurements of the charged jet cross section by the ALICE Collaboration [17], with reduced systematic uncertainties, and are consistent in the common $p_{\mathrm{T}}^{\text {chjet }}$ range. The previous results are superseded by this work.

The measured charged jet cross sections are compared to calculations from the PYTHIA MC model. The ratios of the MC distributions to measured data are shown in the bottom panel. The systematic uncertainty on the data is indicated by a shaded band drawn at unity. The models qualitatively describe the measured cross sections, but fail to reproduce the spectral shape over the entire range of measured jet $p_{\mathrm{T}}^{\text {ch jet }}$. In the high jet transverse momentum range, $p_{\mathrm{T}}^{\text {chjet }}>40 \mathrm{GeV} / c$ both PYTHIA6 tune Perugia-2011 and PYTHIA8 tune Monash describe the data well, whereas at intermediate $p_{\mathrm{T}}^{\text {chjet }}$ the jet cross section is systematically overestimated. The discrepancy is about $30-40 \%$ for $p_{\mathrm{T}}^{\text {chjet }} \approx 10-15 \mathrm{GeV} / c$.

In Fig. 2, the measured cross sections are compared to NLO pQCD calculations with the POWHEG BOX framework, in which the outgoing partons are passed to PYTHIA8 where the subsequent parton shower and hadronization are handled. The UE contribution is subtracted using the method described in Sec. V C in both data and theory calculations. Systematic uncertainties on data and theory

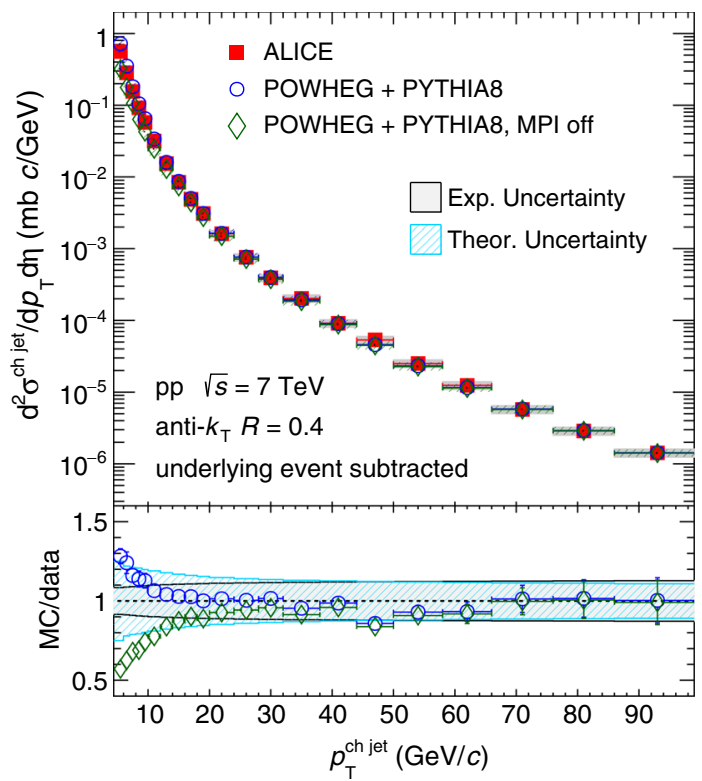

FIG. 2. Top panel: Inclusive charged jet cross section compared to POWHEG+PYTHIA8 NLO pQCD calculations with and without MPI. In data and calculations, the underlying event contribution is subtracted. Bottom panel: Ratio of POWHEG calculations to data. The shaded bands indicate systematic uncertainties on data and theory predictions.

predictions are indicated by shaded bands. The theory systematic uncertainties are related to the choice of scale and PDF as well as the UE subtraction. They are largest at the lowest $p_{\mathrm{T}}^{\text {chjet }}$ and vary between $25 \%$ and $11 \%$. In the jet transverse momentum range $p_{\mathrm{T}}^{\text {chjet }}>7 \mathrm{GeV} / c$, POWHEG+PYTHIA8 (open circles) gives a good description of the data. The spectral shape is reproduced well for $p_{\mathrm{T}}^{\text {chjet }}>20 \mathrm{GeV} / c$. At lower transverse momenta, $5 \leq$ $p_{\mathrm{T}}^{\text {ch jet }}<20 \mathrm{GeV} / c$, the calculations overestimate the measured cross section, but the difference is within the combined experimental and theoretical uncertainties. To study the contribution of soft processes generated in PYTHIA8, the POWHEG+PYTHIA8 calculations were repeated with alternative settings, switching off MPI from PYTHIA8. The calculated jet cross sections without MPI (open diamonds) are smaller than the result with default settings in the range $p_{\mathrm{T}}^{\text {ch jet }}<20 \mathrm{GeV} / c$, and the measured jet cross section is significantly underpredicted for $p_{\mathrm{T}}^{\text {ch jet }}<10 \mathrm{GeV} / c$. The agreement with the data is worse than in the case with MPI. As a further test, we compared the UE activity, measured by the particle $p_{\mathrm{T}}$ density in perpendicular cones, in data and simulations for default and alternative settings. The POWHEG+PYTHIA8 simulations with default settings reproduce the measured UE reasonably well (compare also Ref. [36]), whereas simulations without MPI show a strongly reduced UE $p_{\mathrm{T}}$ density and fail to describe the data. These results indicate a sizable contribution from nonperturbative processes to jet production at 
low $p_{\mathrm{T}}^{\text {ch jet }}$. Comparing the two settings in the simulations, MPI contribute $\sim 50 \%$ to the cross section for $5 \leq p_{\mathrm{T}}^{\text {ch jet }}<$ $10 \mathrm{GeV} / c$ and $\sim 20 \%$ for $p_{\mathrm{T}}^{\text {chjet }} \geq 10 \mathrm{GeV} / c$. In this estimate, a possible additional contribution from initialstate radiation is not taken into account. In a study of low transverse energy clusters in $p \bar{p}$ collisions at $\sqrt{s}=$ $900 \mathrm{GeV}$ [47], the contribution from soft processes to jets with $E_{\mathrm{T}}^{\text {raw }}>5 \mathrm{GeV}$ was evaluated to be $18 \%$, similar in magnitude but lower than our estimate. This difference may be attributed to experimental differences in the definition of the jet energy scale and in the theoretical models, but may also reflect the $\sqrt{s}$ evolution of the probability for MPI, represented by the rise of the UE density observed with collision energy [48].

Next-to-leading-order pQCD calculations overestimate the cross sections for inclusive $\pi^{0}$ and $\eta$ meson production at midrapidity measured in pp collisions at $\sqrt{s}=7 \mathrm{TeV}$ in the $\pi^{0}(\eta)$ transverse momentum range $0.3<p_{\mathrm{T}}<$ $25 \mathrm{GeV} / c\left(0.4<p_{\mathrm{T}}<15 \mathrm{GeV} / c\right)$ by up to a factor of 3 [2]. The jet cross section presented in this work covers a $p_{\mathrm{T}}$ range consistent with Ref. [2], and a consistent PDF set was used for the POWHEG calculations. Since the jet cross section observable depends only weakly on the details of parton fragmentation, the good agreement between data and NLO pQCD calculations for jet cross sections suggests the uncertainty in the parton to hadron fragmentation functions to be the cause for the discrepancy observed for neutral mesons.

The left panel of Fig. 3 presents the measured scaled $p_{\mathrm{T}}$ spectra, $F^{z}$, of charged particles in charged jets reconstructed with a resolution parameter $R=0.4$. The $F^{z}$ distributions are shown for four bins in jet $p_{\mathrm{T}}$ :
$5 \leq p_{\mathrm{T}}^{\text {ch jet }}<10 \mathrm{GeV} / c, \quad 10 \leq p_{\mathrm{T}}^{\text {ch jet }}<15 \mathrm{GeV} / c, \quad 15 \leq$ $p_{\mathrm{T}}^{\text {chjet }}<20 \mathrm{GeV} / c$ and $20 \leq p_{\mathrm{T}}^{\text {ch jet }}<30 \mathrm{GeV} / c$. The spectra span 2 to 3 orders of magnitude. At the lowest $z^{\text {ch }}$, for jets with $p_{\mathrm{T}}^{\text {ch jet }}<10 \mathrm{GeV} / c$ the yield increases to a distinct maximum at $z^{\text {ch }} \approx 0.05$. This nonmonotonic behavior corresponds to the humpbacked plateau at high values of the variable $\xi=\log (1 / z)$ [17,49], which reflects the suppression of low momentum particle production by QCD coherence [50,51]. For jets with $10 \leq p_{\mathrm{T}}^{\text {ch jet }}<15 \mathrm{GeV} / c$ the maximum is less pronounced, and for jets with $15 \leq$ $p_{\mathrm{T}}^{\text {chjet }}<20 \mathrm{GeV} / c$ the yield is roughly constant for $z<0.1$. This reflects a shift of the maximum towards lower $z^{\text {ch }}$ (corresponding to higher $\xi$ ) with increasing $p_{\mathrm{T}}^{\text {ch jet }}$. A similar $p_{\mathrm{T}}^{\text {ch jet }}$ dependence was observed in Ref. [17]. For the highest $z^{\text {ch }}$ bin, the $F^{z}$ distributions for jets with $p_{\mathrm{T}}^{\text {ch jet }}<$ $20 \mathrm{GeV} / c$ show a discontinuous increase, which is strongest for the lowest $p_{\mathrm{T}}^{\text {ch jet }}$ bin. It corresponds to jets with only a single charged constituent, for which $z^{\text {ch }}=1$ by construction. The effect is also observed in the simulations.

An increase of the integral of the distributions with $p_{\mathrm{T}}^{\text {ch jet }}$ is observed, reflecting the rise of particle multiplicity with increasing $p_{\mathrm{T}}^{\text {ch jet }}$ observed in Ref. [17].

In Ref. [17] it was found that the $F^{z}$ distributions measured for leading charged jets in the range $p_{\mathrm{T}}^{\text {ch jet leading }} \geq$ $20 \mathrm{GeV} / c$ are consistent within uncertainties for $z^{\text {ch }}>0.1$, indicating a scaling of charged jet fragmentation with charged jet transverse momentum. For the inclusive charged jet fragmentation distributions in the jet $p_{\mathrm{T}}$ range reported in this work, no such scaling is observed. The shape of the spectra become progressively flatter with

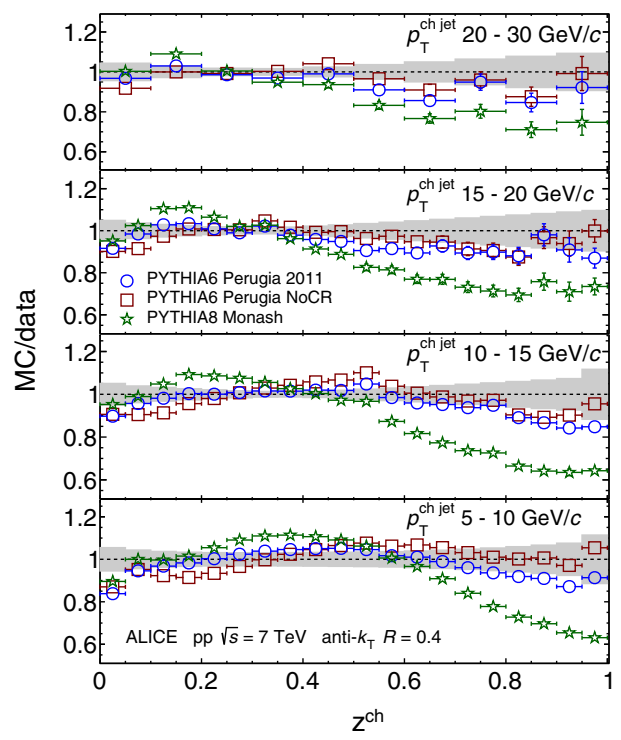

FIG. 3. Left panel: Charged particle scaled $p_{\mathrm{T}}$ spectra $F^{z}\left(z^{\mathrm{ch}}, p_{\mathrm{T}}^{\text {ch jet }}\right)$ for different bins in jet transverse momentum. Right panel: Ratio of MC distributions to data. The shaded band shows the systematic uncertainty on the data drawn at unity. Error bars represent the statistical uncertainties. 
decreasing $p_{\mathrm{T}}^{\text {ch jet }}$. However, comparing $F^{z}$ for the jet $p_{\mathrm{T}}$ bin $15-20 \mathrm{GeV} / c$ and the lowest jet $p_{\mathrm{T}}$ bin, $5-10 \mathrm{GeV} / c$, to $F^{z}$ for the intermediate jet $p_{\mathrm{T}}$ bin, $10-15 \mathrm{GeV} / c$, we observe that the distributions for the two higher bins are more similar to each other than the two lower $p_{\mathrm{T}}$ bins. This may indicate an onset of the scaling behavior. We note that the distribution for inclusive jets with $15 \leq p_{\mathrm{T}}^{\text {chjet }}<$ $20 \mathrm{GeV} / c$ and $20 \leq p_{\mathrm{T}}^{\text {ch jet }}<30 \mathrm{GeV} / c$ exhibit small but significant differences. This indicates that the $z^{\text {ch }}$ scaling reported in Ref. [17] is only fully developed for $p_{\mathrm{T}}^{\text {ch jet }} \geq 20 \mathrm{GeV} / c$.

The measured fragmentation distributions are compared to calculations obtained from the PYTHIA model, and the ratios of the MC distributions to data are presented in the right panel of Fig. 3. The observed trends for the individual tunes are similar for all charged jet $p_{\mathrm{T}}$. The PYTHIA6 tune Perugia-2011 reproduces the fragmentation distributions reasonably well, although there are discrepancies of up to $10-15 \%$ in some kinematic regions. For $p_{\mathrm{T}}^{\text {chjet }} \geq 10 \mathrm{GeV} / c$, the model tends to underpredict the measured yield at high $z^{\text {ch }}$. The PYTHIA8 calculations with the Monash tune exhibit a softer spectrum than the data, overpredicting the fragment yield at intermediate $z^{\text {ch }} \approx$ $0.15-0.4$ and underestimating the rates at high $z^{\mathrm{ch}}$, the discrepancy reaching $\sim 35 \%$ at $z=1$ for the lowest jet $p_{\mathrm{T}}$ bin. The difference between calculations and data at intermediate $z^{\text {ch }}$ is most pronounced at a value of constituent $p_{\mathrm{T}} \approx 2 \mathrm{GeV} / c$ for all four jet $p_{\mathrm{T}}$ intervals. To investigate the observed differences between data and calculations at higher jet $p_{\mathrm{T}}$, we also compared the leading charged jet $F^{z}$ distributions in the range $20 \leq p_{\mathrm{T}}^{\text {ch jet leading }}<$ $80 \mathrm{GeV} / c$ from Ref. [17] to PYTHIA8 simulations. We observe that for $p_{\mathrm{T}}^{\text {ch jet leading }} \geq 40 \mathrm{GeV} / c$ the distributions at intermediate $z^{\text {ch }}$ are well described, whereas the yield at high $z^{\text {ch }}$ is also underestimated for high $p_{\mathrm{T}}^{\text {ch jet leading }}$.

The data are also compared to the PYTHIA6 Perugia NoCR tune [29]. This tune is an attempt to describe the data sets used for the Perugia tunes without invoking color reconnections (CR) [19] between fragmenting partons to model nonperturbative color string interactions. It does not reproduce the data used to constrain the PYTHIA parameter space well. However, for the $F^{z}$ distributions reported in this paper, the calculations agree with the data to within about 10-15\%. In Ref. [52] it was shown that in the PYTHIA8 model, the effect of CR is strong in events with MPI and increases with MPI activity. Hence, the weak effect of color reconnections on the low- $p_{\mathrm{T}}^{\text {chjet }}$ fragmentation distributions in PYTHIA may indicate that these jets are dominantly produced in hard scattering events and from MPI with a few hard outgoing partons, rather than being formed as hadron clusters from the fragmentation of many soft partons combined by the jetfinding algorithm.

\section{SUMMARY}

The inclusive charged jet cross section and jet fragmentation distributions at midrapidity in pp collisions at $\sqrt{s}=$ $7 \mathrm{TeV}$ were measured. The cross section for a resolution parameter $R=0.4$ was reported in the $p_{\mathrm{T}}^{\text {chjet }}$ interval from 5 to $100 \mathrm{GeV} / c$. We studied charged particle fragmentation in charged jets with $5 \leq p_{\mathrm{T}}^{\text {ch jet }}<30 \mathrm{GeV} / c$, extending the range in Ref. [17]. The integral of the fragmentation distributions increases with jet $p_{\mathrm{T}}$, showing an increase of particle multiplicity in jets. The shapes of the distributions become progressively flatter for lower jet $p_{\mathrm{T}}$.

The measurements were compared to PYTHIA calculations. The cross sections are well described by PYTHIA6 and PYTHIA8 for $p_{\mathrm{T}}^{\text {ch jet }}>40 \mathrm{GeV} / c$. At lower $p_{\mathrm{T}}^{\text {ch jet }}$ the PYTHIA tunes studied here fail to describe the shape of the jet spectra and the cross section is systematically overestimated. PYTHIA6 tune Perugia-2011 gives a reasonable description of the fragmentation distributions, whereas the PYTHIA8 tune Monash exhibits a softer spectrum than the data, with significant deviations particularly at high $z^{\text {ch }}$.

The jet cross sections are well described by POWHEG NLO pQCD +PYTHIA8 calculations for the entire measured range of $p_{\mathrm{T}}^{\text {chjet }}$. The simulations indicate a sizable contribution of multiparton interactions to the jet cross section for low $p_{\mathrm{T}}^{\text {ch jet }}$. We found that PYTHIA6 tune NoCR reproduces the measured fragmentation distributions reasonably well in the entire jet $p_{\mathrm{T}}$ range covered by our measurements, possibly indicating that the contribution of events with multiple soft color connected partons to jet production is small in the kinematic regime of our measurement, $p_{\mathrm{T}}^{\text {chjet }}>5 \mathrm{GeV} / c$.

The good agreement between the NLO calculations and the measured jet cross section indicates that the previously observed discrepancies between data and NLO calculations of neutral meson production may be due to the fragmentation functions used in these calculations.

\section{ACKNOWLEDGMENTS}

This was the last work of O. Busch and serves as a memorial to him and his contributions to the ALICE collaboration and the field. The ALICE Collaboration would like to thank all its engineers and technicians for their invaluable contributions to the construction of the experiment and the CERN accelerator teams for the outstanding performance of the LHC complex. The ALICE Collaboration gratefully acknowledges the resources and support provided by all Grid centers and the Worldwide LHC Computing Grid (WLCG) collaboration. The ALICE Collaboration acknowledges the following funding agencies for their support in building and running the ALICE detector: A. I. Alikhanyan National Science Laboratory (Yerevan Physics Institute) Foundation (ANSL), State Committee of Science and World Federation of 
Scientists (WFS), Armenia; Austrian Academy of Sciences and Nationalstiftung für Forschung, Technologie und Entwicklung, Austria; Ministry of Communications and High Technologies, National Nuclear Research Center, Azerbaijan; Conselho Nacional de Desenvolvimento Científico e Tecnológico (CNPq), Universidade Federal do Rio Grande do Sul (UFRGS), Financiadora de Estudos e Projetos (Finep) and Fundaćáo de Amparo à Pesquisa do Estado de São Paulo (FAPESP), Brazil; Ministry of Science $\&$ Technology of China (MSTC), National Natural Science Foundation of China (NSFC) and Ministry of Education of China (MOEC), China; Ministry of Science and Education, Croatia; Centro de Aplicaciones Tecnológicas y Desarrollo Nuclear (CEADEN), Cubaenergía, Cuba; Ministry of Education, Youth and Sports of the Czech Republic, Czech Republic; The Danish Council for Independent Research-Natural Sciences, the Carlsberg Foundation and Danish National Research Foundation (DNRF), Denmark; Helsinki Institute of Physics (HIP), Finland; Commissariat à l'Energie Atomique (CEA) and Institut National de Physique Nucléaire et de Physique des Particules (IN2P3) and Centre National de la Recherche Scientifique (CNRS), France; Bundesministerium für Bildung, Wissenschaft, Forschung und Technologie (BMBF) and GSI Helmholtzzentrum für Schwerionenforschung GmbH, Germany; General Secretariat for Research and Technology, Ministry of Education, Research and Religions, Greece; National Research, Development and Innovation Office, Hungary; Department of Atomic Energy Government of India (DAE), Department of Science and Technology, Government of India (DST), University Grants Commission, Government of India (UGC) and Council of Scientific and Industrial Research (CSIR), India; Indonesian Institute of Science, Indonesia; Centro Fermi-Museo Storico della Fisica e Centro Studi e Ricerche Enrico Fermi and Istituto Nazionale di Fisica Nucleare (INFN), Italy; Institute for Innovative Science and Technology, Nagasaki Institute of
Applied Science (IIST), Japan Society for the Promotion of Science (JSPS) KAKENHI and Japanese Ministry of Education, Culture, Sports, Science and Technology (MEXT), Japan; Consejo Nacional de Ciencia (CONACYT) y Tecnología, through Fondo de Cooperación Internacional en Ciencia y Tecnología (FONCICYT) and Dirección General de Asuntos del Personal Academico (DGAPA), Mexico; Nederlandse Organisatie voor Wetenschappelijk Onderzoek (NWO), Netherlands; The Research Council of Norway, Norway; Commission on Science and Technology for Sustainable Development in the South (COMSATS), Pakistan; Pontificia Universidad Católica del Perú, Peru; Ministry of Science and Higher Education and National Science Centre, Poland; Korea Institute of Science and Technology Information and National Research Foundation of Korea (NRF), Republic of Korea; Ministry of Education and Scientific Research, Institute of Atomic Physics and Romanian National Agency for Science, Technology and Innovation, Romania; Joint Institute for Nuclear Research (JINR), Ministry of Education and Science of the Russian Federation and National Research Centre Kurchatov Institute, Russia; Ministry of Education, Science, Research and Sport of the Slovak Republic, Slovakia; National Research Foundation of South Africa, South Africa; Swedish Research Council (VR) and Knut \& Alice Wallenberg Foundation (KAW), Sweden; European Organization for Nuclear Research, Switzerland; National Science and Technology Development Agency (NSDTA), Suranaree University of Technology (SUT) and Office of the Higher Education Commission under NRU project of Thailand, Thailand; Turkish Atomic Energy Agency (TAEK), Turkey; National Academy of Sciences of Ukraine, Ukraine; Science and Technology Facilities Council (STFC), United Kingdom; National Science Foundation of the United States of America (NSF) and United States Department of Energy, Office of Nuclear Physics (DOE NP), United States of America.
[1] D. d'Enterria, K. J. Eskola, I. Helenius, and H. Paukkunen, Confronting current NLO parton fragmentation functions with inclusive charged-particle spectra at hadron colliders, Nucl. Phys. B883, 615 (2014).

[2] B. Abelev et al. (ALICE Collaboration), Neutral pion and $\eta$ meson production in proton-proton collisions at $\sqrt{s}=$ $0.9 \mathrm{TeV}$ and $\sqrt{s}=7 \mathrm{TeV}$, Phys. Lett. B 717, 162 (2012).

[3] L. E. Gordon and W. Vogelsang, Polarized and unpolarized prompt photon production beyond the leading order, Phys. Rev. D 48, 3136 (1993).

[4] P. Kotko, A. M. Stasto, and M. Strikman, Exploring minijets beyond the leading power, Phys. Rev. D 95, 054009 (2017).
[5] H. Lai, J. Huston, S. Kuhlmann, J. Morfin, F. Olness, J. F. Owens, J. Pumplin, and W. K. Tung (CTEQ Collaboration), Global QCD analysis of parton structure of the nucleon: CTEQ5 parton distributions, Eur. Phys. J. C 12, 375 (2000).

[6] P. Nason, A new method for combining NLO QCD with shower Monte Carlo algorithms, J. High Energy Phys. 11 (2004) 040.

[7] S. Frixione, P. Nason, and C. Oleari, A general framework for implementing NLO calculations in shower Monte Carlo programs: The POWHEG BOX, J. High Energy Phys. 11 (2007) 070. 
[8] S. Alioli, K. Hamilton, P. Nason, C. Oleari, and E. Re, Jet pair production in POWHEG, J. High Energy Phys. 04 (2011) 081.

[9] D. d'Enterria, K. J. Eskola, I. Helenius, and H. Paukkunen, Confronting current NLO parton fragmentation functions with inclusive charged-particle spectra at hadron colliders, Nucl. Phys. B883, 615 (2014).

[10] G. Aad et al. (ATLAS Collaboration), Measurement of the inclusive jet cross-section in proton-proton collisions at $\sqrt{s}=7 \mathrm{TeV}$ using $4.5 \mathrm{fb}^{-1}$ of data with the ATLAS detector, J. High Energy Phys. 02 (2015) 153; Erratum, J. High Energy Phys. 09 (2015) 141(E).

[11] G. Aad et al. (ATLAS Collaboration), Properties of jets measured from tracks in proton-proton collisions at centerof-mass energy $\sqrt{s}=7 \mathrm{TeV}$ with the ATLAS detector, Phys. Rev. D 84, 054001 (2011).

[12] S. Chatrchyan et al. (CMS Collaboration), Measurement of the Inclusive Jet Cross Section in $p p$ Collisions at $\sqrt{s}=7 \mathrm{TeV}$, Phys. Rev. Lett. 107, 132001 (2011).

[13] S. Chatrchyan et al. (CMS Collaboration), Measurements of differential jet cross sections in proton-proton collisions at $\sqrt{s}=7 \mathrm{TeV}$ with the CMS detector, Phys. Rev. D 87, 112002 (2013).

[14] G. Aad et al. (ATLAS Collaboration), Measurement of the jet fragmentation function and transverse profile in protonproton collisions at a center-of-mass energy of $7 \mathrm{TeV}$ with the ATLAS detector, Eur. Phys. J. C 71, 1795 (2011).

[15] G. Aad et al. (ATLAS Collaboration), Measurement of inclusive jet charged-particle fragmentation functions in $\mathrm{Pb}+\mathrm{Pb}$ collisions at $\sqrt{s_{N N}}=2.76 \mathrm{TeV}$ with the ATLAS detector, Phys. Lett. B 739, 320 (2014).

[16] S. Chatrchyan et al. (CMS Collaboration), Measurement of jet fragmentation into charged particles in $p p$ and $\mathrm{PbPb}$ collisions at $\sqrt{s_{N N}}=2.76 \mathrm{TeV}$, J. High Energy Phys. 10 (2012) 087.

[17] B. B. Abelev et al. (ALICE Collaboration), Charged jet cross sections and properties in proton-proton collisions at $\sqrt{s}=7 \mathrm{TeV}$, Phys. Rev. D 91, 112012 (2015).

[18] M. Cacciari, G. P. Salam, and G. Soyez, The Anti-k(t) jet clustering algorithm, J. High Energy Phys. 04 (2008) 063.

[19] G. Gustafson, Multiple interactions, saturation, and final states in $p p$ collisions and DIS, Acta Phys. Pol. B 40, 1981 (2009).

[20] S. Gieseke, C. Rohr, and A. Siodmok, Colour reconnections in Herwig++, Eur. Phys. J. C 72, 2225 (2012).

[21] K. Aamodt et al. (ALICE Collaboration), The ALICE experiment at the CERN LHC, J. Instrum. 3, S08002 (2008).

[22] J. Alme, Y. Andres, H. Appelshauser, S. Bablok, N. Bialas et al., The ALICE TPC, a large 3-dimensional tracking device with fast readout for ultra-high multiplicity events, Nucl. Instrum. Methods Phys. Res., Sect. A 622, 316 (2010).

[23] K. Aamodt et al. (ALICE Collaboration), Alignment of the ALICE inner tracking system with cosmic-ray tracks, J. Instrum. 5, P03003 (2010).

[24] P. Cortese et al. (ALICE Collaboration), ALICE technical design report on forward detectors: FMD, T0 and V0, CERN Report No. CERN-LHCC-2004-025, 2004, https:// cds.cern.ch/record/781854.
[25] B. Abelev et al. (ALICE Collaboration), Measurement of inelastic, single- and double-diffraction cross sections in proton-proton collisions at the LHC with ALICE, Eur. Phys. J. C 73, 2456 (2013).

[26] B. Abelev et al. (ALICE Collaboration), Measurement of the inclusive differential jet cross section in $p p$ collisions at $\sqrt{s}=2.76 \mathrm{TeV}$, Phys. Lett. B 722, 262 (2013).

[27] M. Cacciari and G. P. Salam, Dispelling the $N^{3}$ myth for the $k_{t}$ jet-finder, Phys. Lett. B 641, 57 (2006).

[28] T. Sjostrand, S. Mrenna, and P. Skands, PYTHIA 6.4 physics and manual, J. High Energy Phys. 05 (2006) 026.

[29] P. Z. Skands, Tuning Monte Carlo generators: The Perugia tunes, Phys. Rev. D 82, 074018 (2010).

[30] R. Brun, F. Carminati, and S. Giani, GEANT detector description and simulation tool, CERN Reports No. CERNW5013 and No. CERN-W-5013, https://cds.cern.ch/record/ 1082634

[31] G. Marchesini, B. R. Webber, G. Abbiendi, I. G. Knowles, M. H. Seymour, and L. Stanco, HERWIG: A Monte Carlo event generator for simulating hadron emission reactions with interfering gluons, Comput. Phys. Commun. 67, 465 (1992).

[32] G. Corcella, I. G. Knowles, G. Marchesini, S. Moretti, K. Odagiri, P. Richardson, M. H. Seymour, and B. R. Webber, HERWIG 6: An event generator for hadron emission reactions with interfering gluons (including supersymmetric processes), J. High Energy Phys. 01 (2001) 010.

[33] T. Sjostrand, S. Mrenna, and P.Z. Skands, A brief introduction to PYTHIA 8.1, Comput. Phys. Commun. 178, 852 (2008).

[34] T. Sjostrand and P.Z. Skands, Transverse-momentumordered showers and interleaved multiple interactions, Eur. Phys. J. C 39, 129 (2005).

[35] B. Andersson, G. Gustafson, and B. Soderberg, A general model for jet fragmentation, Z. Phys. C 20, 317 (1983).

[36] P. Skands, S. Carrazza, and J. Rojo, Tuning PYTHIA 8.1: The Monash 2013 tune, Eur. Phys. J. C 74, 3024 (2014).

[37] R. D. Ball, V. Bertone, S. Carrazza, L. Del Debbio, S. Forte, A. Guffanti, N. P. Hartland, and J. Rojo (NNPDF Collaboration), Parton distributions with QED corrections, Nucl. Phys. B877, 290 (2013).

[38] J. Pumplin, D. R. Stump, J. Huston, H. L. Lai, P. M. Nadolsky, and W. K. Tung, New generation of parton distributions with uncertainties from global QCD analysis, J. High Energy Phys. 07 (2002) 012.

[39] M. R. Whalley, D. Bourilkov, and R. C. Group, The Les Houches accord PDFs (LHAPDF) and LHAGLUE, arXiv: hep-ph/0508110.

[40] G. D'Agostini, A Multidimensional unfolding method based on Bayes' theorem, Nucl. Instrum. Methods Phys. Res., Sect. A 362, 487 (1995).

[41] T. Adye, Unfolding algorithms and tests using RooUnfold, arXiv:1105.1160; See also http://hepunx.rl.ac.uk/ adye/ software/unfold/RooUnfold.html.

[42] A. Hocker and V. Kartvelishvili, SVD approach to data unfolding, Nucl. Instrum. Methods Phys. Res., Sect. A 372, 469 (1996).

[43] V. Khachatryan et al. (CMS Collaboration), Strange particle production in $p p$ collisions at $\sqrt{s}=0.9$ and $7 \mathrm{TeV}$, J. High Energy Phys. 05 (2011) 064. 
[44] A. Karneyeu, L. Mijovic, S. Prestel, and P. Z. Skands, MCPLOTS: A particle physics resource based on volunteer computing, Eur. Phys. J. C 74, 2714 (2014).

[45] P. Skands et al., https://mcplots.cern.ch.

[46] B. Abelev et al. (ALICE Collaboration), Centrality dependence of charged particle production at large transverse momentum in $\mathrm{Pb}-\mathrm{Pb}$ Collisions at $\sqrt{s_{N N}}=2.76 \mathrm{TeV}$, Phys. Lett. B 720, 52 (2013).

[47] C. Albajar et al. (UA1 Collaboration), Production of low transverse energy clusters in anti- $p p$ collisions at $s * *(1 / 2)=0.2-\mathrm{TeV}$ to $0.9-\mathrm{TeV}$ and their interpretation in terms of QCD jets, Nucl. Phys. B309, 405 (1988).

[48] B. Abelev et al. (ALICE Collaboration), Underlying event measurements in $p p$ collisions at $\sqrt{s}=0.9$ and $7 \mathrm{TeV}$ with the ALICE experiment at the LHC, J. High Energy Phys. 07 (2012) 116.

[49] D. Acosta et al. (CDF Collaboration), Momentum distribution of charged particles in jets in dijet events in $p \bar{p}$ collisions at $\sqrt{s}=1.8 \mathrm{TeV}$ and comparisons to perturbative QCD predictions, Phys. Rev. D 68, 012003 (2003).

[50] B. Ermolaev and V.S. Fadin, Log-Log asymptotic form of exclusive cross-sections in quantum chromodynamics, JETP Lett. 33, 269 (1981).

[51] A. H. Mueller, On the multiplicity of hadrons in QCD jets, Phys. Lett. 104B, 161 (1981).

[52] A. O. Velasquez, P. Christiansen, E. C. Flores, I. M. Cervantes, and G. Paić, Color Reconnection and Flowlike Patterns in $p p$ Collisions, Phys. Rev. Lett. 111, 042001 (2013).

S. Acharya, ${ }^{139}$ D. Adamová, ${ }^{93}$ A. Adler, ${ }^{74}$ J. Adolfsson, ${ }^{80}$ M. M. Aggarwal, ${ }^{98}$ G. Aglieri Rinella, ${ }^{34}$ M. Agnello, ${ }^{31}$ N. Agrawal, ${ }^{48}$ Z. Ahammed, ${ }^{139}$ S. U. Ahn, ${ }^{76}$ S. Aiola, ${ }^{144}$ A. Akindinov,${ }^{64}$ M. Al-Turany, ${ }^{104}$ S. N. Alam, ${ }^{139}$ D. S. D. Albuquerque, ${ }^{121}$ D. Aleksandrov, ${ }^{87}$ B. Alessandro, ${ }^{58}$ R. Alfaro Molina, ${ }^{72}$ Y. Ali, ${ }^{15}$ A. Alici, ${ }^{10,27,53}$ A. Alkin, ${ }^{2}$ J. Alme, ${ }^{22}$ T. Alt, ${ }^{69}$ L. Altenkamper, ${ }^{22}$ I. Altsybeev, ${ }^{111}$ M. N. Anaam, ${ }^{6}$ C. Andrei, ${ }^{47}$ D. Andreou, ${ }^{34}$ H. A. Andrews, ${ }^{108}$ A. Andronic, ${ }^{142,104}$ M. Angeletti, ${ }^{34}$ V. Anguelov, ${ }^{102}$ C. Anson, ${ }^{16}$ T. Antičici,${ }^{105}$ F. Antinori, ${ }^{56}$ P. Antonioli,${ }^{53}$ R. Anwar, ${ }^{125}$ N. Apadula ${ }^{79}$ L. Aphecetche, ${ }^{113}$ H. Appelshäuser, ${ }^{69}$ S. Arcelli, ${ }^{27}$ R. Arnaldi, ${ }^{58}$ O. W. Arnold, ${ }^{103,116}$ I. C. Arsene, ${ }^{21}$ M. Arslandok, ${ }^{102}$ A. Augustinus, ${ }^{34}$ R. Averbeck, ${ }^{104}$ M. D. Azmi,${ }^{17}$ A. Badală,${ }^{55}$ Y. W. Baek,${ }^{60,40}$ S. Bagnasco, ${ }^{58}$ R. Bailhache, ${ }^{69}$ R. Bala, ${ }^{99}$ A. Baldisseri, ${ }^{135}$ M. Ball,${ }^{42}$ R. C. Baral,${ }^{85}$ A. M. Barbano, ${ }^{26}$ R. Barbera,${ }^{28}$ F. Barile, ${ }^{52}$ L. Barioglio, ${ }^{26}$ G. G. Barnaföldi, ${ }^{143}$ L. S. Barnby, ${ }^{92}$ V. Barret,${ }^{132}$ P. Bartalini, ${ }^{6}$ K. Barth,${ }^{34}$ E. Bartsch, ${ }^{69}$ N. Bastid,${ }^{132}$ S. Basu, ${ }^{141}$ G. Batigne, ${ }^{113}$ B. Batyunya ${ }^{75}$ P. C. Batzing, ${ }^{21}$ J. L. Bazo Alba ${ }^{109}$ I. G. Bearden, ${ }^{88}$ H. Beck, ${ }^{102}$ C. Bedda,${ }^{63}$ N. K. Behera ${ }^{60}$ I. Belikov, ${ }^{134}$ F. Bellini,${ }^{34}$ H. Bello Martinez,${ }^{44}$ R. Bellwied, ${ }^{125}$ L. G. E. Beltran, ${ }^{119}$ V. Belyaev, ${ }^{91}$ G. Bencedi, ${ }^{143}$ S. Beole, ${ }^{26}$ A. Bercuci,${ }^{47}$ Y. Berdnikov,${ }^{96}$ D. Berenyi, ${ }^{143}$ R. A. Bertens, ${ }^{128}$ D. Berzano, ${ }^{58,34}$ L. Betev, ${ }^{34}$ P. P. Bhaduri, ${ }^{139}$ A. Bhasin, ${ }^{99}$ I. R. Bhat,${ }^{99}$ H. Bhatt, ${ }^{48}$ B. Bhattacharjee, ${ }^{41}$ J. Bhom, ${ }^{17}$ A. Bianchi, ${ }^{26}$ L. Bianchi, ${ }^{125}$ N. Bianchi, ${ }^{51}$ J. Bielčík,${ }^{37}$ J. Bielčíková, ${ }^{93}$ A. Bilandzic, ${ }^{103,116}$ G. Biro, ${ }^{143}$ R. Biswas, ${ }^{3}$ S. Biswas, ${ }^{3}$ J. T. Blair, ${ }^{118}$ D. Blau, ${ }^{87}$ C. Blume,${ }^{69}$ G. Boca,,${ }^{137}$ F. Bock, ${ }^{34}$ A. Bogdanov,${ }^{91}$ L. Boldizsár, ${ }^{143}$ M. Bombara,${ }^{38}$ G. Bonomi, ${ }^{138}$ M. Bonora, ${ }^{34}$ H. Borel, ${ }^{135}$ A. Borissov ${ }^{142}$ M. Borri, ${ }^{127}$ E. Botta ${ }^{26}$ C. Bourjau, ${ }^{88}$ L. Bratrud,${ }^{69}$ P. Braun-Munzinger, ${ }^{104}$ M. Bregant, ${ }^{120}$ T. A. Broker, ${ }^{69}$ M. Broz ${ }^{37}$ E. J. Brucken, ${ }^{43}$ E. Bruna,${ }^{58}$ G. E. Bruno, ${ }^{34,33}$ D. Budnikov, ${ }^{106}$ H. Buesching, ${ }^{69}$ S. Bufalino, ${ }^{31}$ P. Buhler, ${ }^{112}$ P. Buncic ${ }^{34}$ O. Busch,${ }^{131,}$ Z. Buthelezi, ${ }^{73}$ J. B. Butt, ${ }^{15}$ J. T. Buxton, ${ }^{95}$ J. Cabala,${ }^{115}$ D. Caffarri, ${ }^{89}$ H. Caines, ${ }^{144}$ A. Caliva, ${ }^{104}$ E. Calvo Villar, ${ }^{109}$ R. S. Camacho, ${ }^{44}$ P. Camerini, ${ }^{25}$ A. A. Capon, ${ }^{112}$ W. Carena,${ }^{34}$ F. Carnesecchi, ${ }^{10,27}$

J. Castillo Castellanos, ${ }^{135}$ A. J. Castro, ${ }^{128}$ E. A. R. Casula, ${ }^{54}$ C. Ceballos Sanchez, ${ }^{8}$ S. Chandra, ${ }^{139}$ B. Chang, ${ }^{126}$ W. Chang, ${ }^{6}$ S. Chapeland,${ }^{34}$ M. Chartier, ${ }^{127}$ S. Chattopadhyay, ${ }^{139}$ S. Chattopadhyay, ${ }^{107}$ A. Chauvin, ${ }^{24}$ C. Cheshkov, ${ }^{133}$ B. Cheynis, ${ }^{133}$ V. Chibante Barroso, ${ }^{34}$ D. D. Chinellato, ${ }^{121}$ S. Cho, ${ }^{60}$ P. Chochula,${ }^{34}$ T. Chowdhury, ${ }^{132}$ P. Christakoglou, ${ }^{89}$

C. H. Christensen ${ }^{88}$ P. Christiansen, ${ }^{80}$ T. Chujo, ${ }^{131}$ S. U. Chung, ${ }^{18}$ C. Cicalo,${ }^{54}$ L. Cifarelli, ${ }^{10,27}$ F. Cindolo,${ }^{53}$ J. Cleymans, ${ }^{124}$ F. Colamaria, ${ }^{52}$ D. Colella, ${ }^{52}$ A. Collu, ${ }^{79}$ M. Colocci, ${ }^{27}$ M. Concas,${ }^{58}$ G. Conesa Balbastre, ${ }^{78}$ Z. Conesa del Valle, ${ }^{61}$ J. G. Contreras, ${ }^{37}$ T. M. Cormier, ${ }^{94}$ Y. Corrales Morales, ${ }^{58}$ P. Cortese, ${ }^{32}$ M. R. Cosentino, ${ }^{122}$ F. Costa, ${ }^{34}$ S. Costanza, ${ }^{137}$ J. Crkovská, ${ }^{61}$ P. Crochet, ${ }^{132}$ E. Cuautle,${ }^{70}$ L. Cunqueiro,${ }^{94,142}$ T. Dahms,${ }^{103,116}$ A. Dainese, ${ }^{56}$ F. P. A. Damas, ${ }^{113,135}$ S. Dani, ${ }^{66}$ M. C. Danisch, ${ }^{102}$ A. Danu, ${ }^{68}$ D. Das,${ }^{107}$ I. Das, ${ }^{107}$ S. Das, ${ }^{3}$ A. Dash,${ }^{85}$ S. Dash,${ }^{48}$ S. De,${ }^{49}$ A. De Caro, ${ }^{30}$ G. de Cataldo, ${ }^{52}$ C. de Conti, ${ }^{120}$ J. de Cuveland, ${ }^{39}$ A. De Falco, ${ }^{24}$ D. De Gruttola, ${ }^{10,30}$ N. De Marco, ${ }^{58}$ S. De Pasquale, ${ }^{30}$ R. D. De Souza, ${ }^{121}$ H. F. Degenhardt, ${ }^{120}$ A. Deisting, ${ }^{104,102}$ A. Deloff, ${ }^{84}$ S. Delsanto, ${ }^{26}$ C. Deplano, ${ }^{89}$ P. Dhankher,${ }^{48}$ D. Di Bari, ${ }^{33}$ A. Di Mauro, ${ }^{34}$ B. Di Ruzza, ${ }^{56}$ R. A. Diaz, ${ }^{8}$ T. Dietel, ${ }^{124}$ P. Dillenseger,${ }^{69}$ Y. Ding, ${ }^{6}$ R. Divià, ${ }^{34} \varnothing$. Djuvsland,${ }^{22}$ A. Dobrin,${ }^{34}$ D. Domenicis Gimenez, ${ }^{120}$ B. Dönigus, ${ }^{69}$ O. Dordic, ${ }^{21}$ L. V. R. Doremalen, ${ }^{63}$ A. K. Dubey, ${ }^{139}$ A. Dubla,${ }^{104}$ L. Ducroux, ${ }^{133}$ S. Dudi, ${ }^{98}$ A. K. Duggal,${ }^{98}$ M. Dukhishyam, ${ }^{85}$ P. Dupieux, ${ }^{132}$ R. J. Ehlers, ${ }^{144}$ D. Elia, ${ }^{52}$ E. Endress, ${ }^{109}$ H. Engel,${ }^{74}$ E. Epple, ${ }^{144}$ B. Erazmus, ${ }^{113}$ F. Erhardt,${ }^{97}$ M. R. Ersdal, ${ }^{22}$ B. Espagnon, ${ }^{61}$ G. Eulisse, ${ }^{34}$ J. Eum, ${ }^{18}$ D. Evans,${ }^{108}$ S. Evdokimov, ${ }^{90}$ L. Fabbietti, ${ }^{103,116}$ M. Faggin, ${ }^{29}$ J. Faivre, ${ }^{78}$ A. Fantoni, ${ }^{51}$ M. Fasel, ${ }^{94}$ L. Feldkamp, ${ }^{142}$ A. Feliciello, ${ }^{58}$ 
G. Feofilov, ${ }^{111}$ A. Fernández Téllez, ${ }^{44}$ A. Ferretti, ${ }^{26}$ A. Festanti, ${ }^{34}$ V. J. G. Feuillard, ${ }^{102}$ J. Figiel, ${ }^{117}$ M. A. S. Figueredo, ${ }^{120}$ S. Filchagin, ${ }^{106}$ D. Finogeev, ${ }^{62}$ F. M. Fionda, ${ }^{22}$ G. Fiorenza, ${ }^{52}$ F. Flor, ${ }^{125}$ M. Floris, ${ }^{34}$ S. Foertsch, ${ }^{73}$ P. Foka,${ }^{104}$ S. Fokin, ${ }^{87}$

E. Fragiacomo, ${ }^{59}$ A. Francescon, ${ }^{34}$ A. Francisco, ${ }^{113}$ U. Frankenfeld, ${ }^{104}$ G. G. Fronze, ${ }^{26}$ U. Fuchs, ${ }^{34}$ C. Furget, ${ }^{78}$ A. Furs,${ }^{62}$ M. Fusco Girard, ${ }^{30}$ J. J. Gaardhøje, ${ }^{88}$ M. Gagliardi, ${ }^{26}$ A. M. Gago, ${ }^{109}$ K. Gajdosova, ${ }^{88}$ M. Gallio, ${ }^{26}$ C. D. Galvan, ${ }^{119}$

P. Ganoti, ${ }^{83}$ C. Garabatos, ${ }^{104}$ E. Garcia-Solis, ${ }^{11}$ K. Garg, ${ }^{28}$ C. Gargiulo, ${ }^{34}$ P. Gasik, ${ }^{116,103}$ E. F. Gauger, ${ }^{118}$

M. B. Gay Ducati, ${ }^{71}$ M. Germain, ${ }^{113}$ J. Ghosh, ${ }^{107}$ P. Ghosh, ${ }^{139}$ S. K. Ghosh, ${ }^{3}$ P. Gianotti, ${ }^{51}$ P. Giubellino, ${ }^{104,58}$ P. Giubilato, ${ }^{29}$

P. Glässel,${ }^{102}$ D. M. Goméz Coral, ${ }^{72}$ A. Gomez Ramirez ${ }^{74}$ V. Gonzalez, ${ }^{104}$ P. González-Zamora, ${ }^{44}$ S. Gorbunov, ${ }^{39}$

L. Görlich, ${ }^{117}$ S. Gotovac, ${ }^{35}$ V. Grabski, ${ }^{72}$ L. K. Graczykowski, ${ }^{140}$ K. L. Graham, ${ }^{108}$ L. Greiner, ${ }^{79}$ A. Grelli, ${ }^{63}$ C. Grigoras,${ }^{34}$ V. Grigoriev, ${ }^{91}$ A. Grigoryan, ${ }^{1}$ S. Grigoryan, ${ }^{75}$ J. M. Gronefeld, ${ }^{104}$ F. Grosa,${ }^{31}$ J. F. Grosse-Oetringhaus, ${ }^{34}$ R. Grosso, ${ }^{104}$ R. Guernane, ${ }^{78}$ B. Guerzoni, ${ }^{27}$ M. Guittiere, ${ }^{113}$ K. Gulbrandsen, ${ }^{88}$ T. Gunji, ${ }^{130}$ A. Gupta, ${ }^{99}$ R. Gupta, ${ }^{99}$ I. B. Guzman, ${ }^{44}$ R. Haake, ${ }^{34,144}$ M. K. Habib, ${ }^{104}$ C. Hadjidakis, ${ }^{61}$ H. Hamagaki, ${ }^{81}$ G. Hamar, ${ }^{143}$ M. Hamid, ${ }^{6}$ J. C. Hamon, ${ }^{134}$ R. Hannigan, ${ }^{118}$

M. R. Haque, ${ }^{63}$ A. Harlenderova, ${ }^{104}$ J. W. Harris, ${ }^{144}$ A. Harton, ${ }^{11}$ H. Hassan, ${ }^{78}$ D. Hatzifotiadou, ${ }^{53,10}$ S. Hayashi, ${ }^{130}$

S. T. Heckel, ${ }^{69}$ E. Hellbär, ${ }^{69}$ H. Helstrup ${ }^{36}$ A. Herghelegiu, ${ }^{47}$ E. G. Hernandez,${ }^{44}$ G. Herrera Corral, ${ }^{9}$ F. Herrmann, ${ }^{142}$

K. F. Hetland, ${ }^{36}$ T. E. Hilden, ${ }^{43}$ H. Hillemanns,${ }^{34}$ C. Hills, ${ }^{127}$ B. Hippolyte,${ }^{134}$ B. Hohlweger, ${ }^{103}$ D. Horak, ${ }^{37}$ S. Hornung, ${ }^{104}$

R. Hosokawa, ${ }^{78,131}$ J. Hota, ${ }^{66}$ P. Hristov, ${ }^{34}$ C. Huang, ${ }^{61}$ C. Hughes,${ }^{128}$ P. Huhn,${ }^{69}$ T. J. Humanic, ${ }^{95}$ H. Hushnud, ${ }^{107}$

N. Hussain, ${ }^{41}$ T. Hussain, ${ }^{17}$ D. Hutter, ${ }^{39}$ D. S. Hwang, ${ }^{19}$ J. P. Iddon, ${ }^{127}$ S. A. Iga Buitron, ${ }^{70}$ R. Ilkaev, ${ }^{106}$ M. Inaba, ${ }^{131}$

M. Ippolitov ${ }^{87}$ M. S. Islam, ${ }^{107}$ M. Ivanov, ${ }^{104}$ V. Ivanov, ${ }^{96}$ V. Izucheev,${ }^{90}$ B. Jacak ${ }^{79}$ N. Jacazio, ${ }^{27}$ P. M. Jacobs, ${ }^{79}$

M. B. Jadhav, ${ }^{48}$ S. Jadlovska, ${ }^{115}$ J. Jadlovsky, ${ }^{115}$ S. Jaelani, ${ }^{63}$ C. Jahnke, ${ }^{120,116}$ M. J. Jakubowska, ${ }^{140}$ M. A. Janik, ${ }^{140}$

C. Jena, ${ }^{85}$ M. Jercic, ${ }^{97}$ O. Jevons, ${ }^{108}$ R. T. Jimenez Bustamante, ${ }^{104}$ M. Jin, ${ }^{125}$ P. G. Jones, ${ }^{108}$ A. Jusko, ${ }^{108}$ P. Kalinak,${ }^{65}$ A. Kalweit, ${ }^{34}$ J. H. Kang, ${ }^{145}$ V. Kaplin, ${ }^{91}$ S. Kar, ${ }^{6}$ A. Karasu Uysal, ${ }^{77}$ O. Karavichev, ${ }^{62}$ T. Karavicheva, ${ }^{62}$ P. Karczmarczyk, ${ }^{34}$ E. Karpechev, ${ }^{62}$ U. Kebschull, ${ }^{74}$ R. Keidel, ${ }^{46}$ D. L. D. Keijdener, ${ }^{63}$ M. Keil ${ }^{34}$ B. Ketzer, ${ }^{42}$ Z. Khabanova, ${ }^{89}$ A. M. Khan, ${ }^{6}$ S. Khan, ${ }^{17}$ S. A. Khan, ${ }^{139}$ A. Khanzadeev, ${ }^{96}$ Y. Kharlov, ${ }^{90}$ A. Khatun, ${ }^{17}$ A. Khuntia, ${ }^{49}$ M. M. Kielbowicz, ${ }^{117}$ B. Kileng,${ }^{36}$ B. Kim, ${ }^{131}$ D. Kim, ${ }^{145}$ D. J. Kim, ${ }^{126}$ E. J. Kim, ${ }^{13}$ H. Kim, ${ }^{145}$ J. S. Kim,${ }^{40}$ J. Kim, ${ }^{102}$ M. Kim, ${ }^{60,102}$ S. Kim, ${ }^{19}$ T. Kim, ${ }^{145}$ T. Kim, ${ }^{145}$ K. Kindra, ${ }^{98}$ S. Kirsch, ${ }^{39}$ I. Kisel, ${ }^{39}$ S. Kiselev, ${ }^{64}$ A. Kisiel, ${ }^{140}$ J. L. Klay, ${ }^{5}$ C. Klein, ${ }^{69}$ J. Klein, ${ }^{34,58}$

C. Klein-Bösing, ${ }^{142}$ S. Klewin, ${ }^{102}$ A. Kluge, ${ }^{34}$ M. L. Knichel, ${ }^{34}$ A. G. Knospe,${ }^{125}$ C. Kobdaj,,${ }^{114}$ M. Kofarago, ${ }^{143}$ M. K. Köhler, ${ }^{102}$ T. Kollegger, ${ }^{104}$ N. Kondratyeva, ${ }^{91}$ E. Kondratyuk, ${ }^{90}$ A. Konevskikh, ${ }^{62}$ P. J. Konopka, ${ }^{34}$

M. Konyushikhin, ${ }^{141}$ L. Koska, ${ }^{15}$ O. Kovalenko, ${ }^{84}$ V. Kovalenko, ${ }^{111}$ M. Kowalski, ${ }^{117}$ I. Králik, ${ }^{65}$ A. Kravčáková, ${ }^{38}$ L. Kreis,${ }^{104}$ M. Krivda, ${ }^{108,65}$ F. Krizek, ${ }^{93}$ M. Krüger, ${ }^{69}$ E. Kryshen,${ }^{96}$ M. Krzewicki, ${ }^{39}$ A. M. Kubera, ${ }^{95}$ V. Kučera, ${ }^{93,60}$ C. Kuhn, ${ }^{134}$ P. G. Kuijer, ${ }^{89}$ J. Kumar, ${ }^{48}$ L. Kumar, ${ }^{98}$ S. Kumar, ${ }^{48}$ S. Kundu, ${ }^{85}$ P. Kurashvili, ${ }^{84}$ A. Kurepin, ${ }^{62}$ A. B. Kurepin, ${ }^{62}$ S. Kushpil ${ }^{93}$ J. Kvapil, ${ }^{108}$ M. J. Kweon, ${ }^{60}$ Y. Kwon, ${ }^{145}$ S. L. La Pointe, ${ }^{39}$ P. La Rocca, ${ }^{28}$ Y. S. Lai, ${ }^{79}$ I. Lakomov, ${ }^{34}$ R. Langoy, ${ }^{123}$ K. Lapidus, ${ }^{144}$ A. Lardeux, ${ }^{21}$ P. Larionov,${ }^{51}$ E. Laudi, ${ }^{34}$ R. Lavicka, ${ }^{37}$ R. Lea, ${ }^{25}$ L. Leardini,${ }^{102}$ S. Lee, ${ }^{145}$ F. Lehas, ${ }^{89}$ S. Lehner, ${ }^{12}$ J. Lehrbach, ${ }^{39}$ R. C. Lemmon, ${ }^{92}$ I. León Monzón, ${ }^{119}$ P. Lévai, ${ }^{143}$ X. Li, ${ }^{12}$ X. L. Li, ${ }^{6}$ J. Lien, ${ }^{123}$ R. Lietava, ${ }^{108}$ B. Lim, ${ }^{18}$ S. Lindal, ${ }^{21}$ V. Lindenstruth, ${ }^{39}$ S. W. Lindsay, ${ }^{127}$ C. Lippmann, ${ }^{104}$ M. A. Lisa, ${ }^{95}$ V. Litichevskyi, ${ }^{43}$ A. Liu, ${ }^{79}$ H. M. Ljunggren, ${ }^{80}$ W. J. Llope, ${ }^{141}$ D. F. Lodato,${ }^{63}$ V. Loginov, ${ }^{91}$ C. Loizides,${ }^{94,79}$ P. Loncar,${ }^{35}$ X. Lopez, ${ }^{132}$ E. López Torres, ${ }^{8}$ P. Luettig, ${ }^{69}$ J. R. Luhder, ${ }^{142}$ M. Lunardon, ${ }^{29}$ G. Luparello, ${ }^{59}$ M. Lupi ${ }^{34}$ A. Maevskaya, ${ }^{62}$ M. Mager, ${ }^{34}$

S. M. Mahmood, ${ }^{21}$ A. Maire, ${ }^{134}$ R. D. Majka, ${ }^{144}$ M. Malaev, ${ }^{96}$ Q. W. Malik, ${ }^{21}$ L. Malinina, ${ }^{75 \S,}$ D. Mal'Kevich, ${ }^{64}$

P. Malzacher, ${ }^{104}$ A. Mamonov, ${ }^{106}$ V. Manko, ${ }^{87}$ F. Manso, ${ }^{132}$ V. Manzari, ${ }^{52}$ Y. Mao, ${ }^{6}$ M. Marchisone, ${ }^{133,73,129}$ J. Mareš ${ }^{67}$

G. V. Margagliotti, ${ }^{25}$ A. Margotti, ${ }^{53}$ J. Margutti, ${ }^{63}$ A. Marín, ${ }^{104}$ C. Markert, ${ }^{118}$ M. Marquard, ${ }^{69}$ N. A. Martin, ${ }^{104}$

P. Martinengo, ${ }^{34}$ J. L. Martinez, ${ }^{125}$ M. I. Martínez, ${ }^{44}$ G. Martínez García, ${ }^{113}$ M. Martinez Pedreira, ${ }^{34}$ S. Masciocchi, ${ }^{104}$ M. Masera, ${ }^{26}$ A. Masoni, ${ }^{54}$ L. Massacrier,${ }^{61}$ E. Masson, ${ }^{113}$ A. Mastroserio, ${ }^{52,136}$ A. M. Mathis, ${ }^{116,103}$ P. F. T. Matuoka, ${ }^{120}$ A. Matyja ${ }^{117,128}$ C. Mayer,${ }^{117}$ M. Mazzilli, ${ }^{33}$ M. A. Mazzoni,${ }^{57}$ F. Meddi, ${ }^{23}$ Y. Melikyan, ${ }^{91}$ A. Menchaca-Rocha, ${ }^{72}$ E. Meninno, ${ }^{30}$ J. Mercado Pérez, ${ }^{102}$ M. Meres, ${ }^{14}$ S. Mhlanga, ${ }^{124}$ Y. Miake, ${ }^{131}$ L. Micheletti, ${ }^{26}$ M. M. Mieskolainen, ${ }^{43}$

D. L. Mihaylov, ${ }^{103}$ K. Mikhaylov, ${ }^{64,75}$ A. Mischke, ${ }^{63}$ A. N. Mishra, ${ }^{70}$ D. Miśkowiec, ${ }^{104}$ J. Mitra ${ }^{139}$ C. M. Mitu, ${ }^{68}$ N. Mohammadi, ${ }^{34}$ A. P. Mohanty ${ }^{63}$ B. Mohanty, ${ }^{85}$ M. Mohisin Khan, ${ }^{17 \|}$ D. A. Moreira De Godoy, ${ }^{142}$ L. A. P. Moreno, ${ }^{44}$ S. Moretto, ${ }^{29}$ A. Morreale, ${ }^{113}$ A. Morsch, ${ }^{34}$ T. Mrnjavac, ${ }^{34}$ V. Muccifora, ${ }^{51}$ E. Mudnic,${ }^{35}$ D. Mühlheim, ${ }^{142}$ S. Muhuri, ${ }^{139}$ M. Mukherjee, ${ }^{3}$ J. D. Mulligan, ${ }^{144}$ M. G. Munhoz, ${ }^{120}$ K. Münning, ${ }^{42}$ M. I. A. Munoz, ${ }^{79}$ R. H. Munzer, ${ }^{69}$ H. Murakami, ${ }^{130}$ S. Murray,${ }^{73}$ L. Musa, ${ }^{34}$ J. Musinsky, ${ }^{65}$ C. J. Myers, ${ }^{125}$ J. W. Myrcha, ${ }^{140}$ B. Naik, ${ }^{48}$ R. Nair,${ }^{84}$ B. K. Nandi, ${ }^{48}$ R. Nania, ${ }^{53,10}$ E. Nappi,${ }^{52}$ A. Narayan, ${ }^{48}$ M. U. Naru, ${ }^{15}$ A. F. Nassirpour, ${ }^{80}$ H. Natal da Luz ${ }^{120}$ C. Nattrass, ${ }^{128}$ S. R. Navarro, ${ }^{44}$ K. Nayak, ${ }^{85}$ 
R. Nayak ${ }^{48}$ T. K. Nayak, ${ }^{139}$ S. Nazarenko, ${ }^{106}$ R. A. Negrao De Oliveira, ${ }^{69,34}$ L. Nellen, ${ }^{70}$ S. V. Nesbo, ${ }^{36}$ G. Neskovic, ${ }^{39}$ F. Ng, ${ }^{125}$ M. Nicassio, ${ }^{104}$ J. Niedziela, ${ }^{140,34}$ B. S. Nielsen, ${ }^{88}$ S. Nikolaev,${ }^{87}$ S. Nikulin, ${ }^{87}$ V. Nikulin, ${ }^{96}$ F. Noferini, ${ }^{10,53}$ P. Nomokonov, ${ }^{75}$ G. Nooren, ${ }^{63}$ J. C. C. Noris, ${ }^{44}$ J. Norman,${ }^{78}$ A. Nyanin,${ }^{87}$ J. Nystrand,${ }^{22}$ H. Oh, ${ }^{145}$ A. Ohlson, ${ }^{102}$ J. Oleniacz, ${ }^{140}$ A. C. Oliveira Da Silva, ${ }^{120}$ M. H. Oliver,${ }^{144}$ J. Onderwaater, ${ }^{104}$ C. Oppedisano, ${ }^{58}$ R. Orava, ${ }^{43}$ M. Oravec,${ }^{115}$ A. Ortiz Velasquez ${ }^{70}$ A. Oskarsson, ${ }^{80}$ J. Otwinowski, ${ }^{117}$ K. Oyama, ${ }^{81}$ Y. Pachmayer, ${ }^{102}$ V. Pacik, ${ }^{88}$ D. Pagano, ${ }^{138}$ G. Paić,${ }^{70}$ P. Palni, ${ }^{6}$ J. Pan, ${ }^{141}$ A. K. Pandey, ${ }^{48}$ S. Panebianco,${ }^{135}$ V. Papikyan, ${ }^{1}$ P. Pareek, ${ }^{49}$ J. Park, ${ }^{60}$ J. E. Parkkila, ${ }^{126}$ S. Parmar, ${ }^{98}$ A. Passfeld, ${ }^{142}$ S. P. Pathak, ${ }^{125}$ R. N. Patra, ${ }^{139}$ B. Paul,${ }^{58}$ H. Pei,${ }^{6}$ T. Peitzmann,${ }^{63}$ X. Peng, ${ }^{6}$ L. G. Pereira, ${ }^{71}$ H. Pereira Da Costa, ${ }^{135}$ D. Peresunko, ${ }^{87}$ E. Perez Lezama, ${ }^{69}$ V. Peskov, ${ }^{69}$ Y. Pestov, ${ }^{4}$ V. Petráček, ${ }^{37}$ M. Petrovici, ${ }^{47}$ C. Petta, ${ }^{28}$ R. P. Pezzi, ${ }^{71}$ S. Piano, ${ }^{59}$ M. Pikna, ${ }^{14}$ P. Pillot,${ }^{13}$ L. O. D. L. Pimentel, ${ }^{88}$ O. Pinazza, ${ }^{53,34}$ L. Pinsky, ${ }^{125}$ S. Pisano, ${ }^{51}$ D. B. Piyarathna, ${ }^{125}$ M. Płoskoń, ${ }^{79}$ M. Planinic,${ }^{97}$ F. Pliquett, ${ }^{69}$ J. Pluta, ${ }^{140}$ S. Pochybova, ${ }^{143}$ P. L. M. Podesta-Lerma, ${ }^{119}$ M. G. Poghosyan,${ }^{94}$ B. Polichtchouk, ${ }^{90}$ N. Poljak, ${ }^{97}$ W. Poonsawat, ${ }^{114}$ A. Pop, ${ }^{47}$ H. Poppenborg, ${ }^{142}$

S. Porteboeuf-Houssais, ${ }^{132}$ V. Pozdniakov, ${ }^{75}$ S. K. Prasad, ${ }^{3}$ R. Preghenella, ${ }^{53}$ F. Prino, ${ }^{58}$ C. A. Pruneau, ${ }^{141}$ I. Pshenichnov,${ }^{62}$ M. Puccio, ${ }^{26}$ V. Punin, ${ }^{106}$ J. Putschke, ${ }^{141}$ S. Raha,${ }^{3}$ S. Rajput, ${ }^{99}$ J. Rak, ${ }^{126}$ A. Rakotozafindrabe, ${ }^{135}$ L. Ramello, ${ }^{32}$ F. Rami, ${ }^{134}$ R. Raniwala, ${ }^{100}$ S. Raniwala, ${ }^{100}$ S. S. Räsänen, ${ }^{43}$ B. T. Rascanu, ${ }^{69}$ R. Rath, ${ }^{49}$ V. Ratza, ${ }^{42}$ I. Ravasenga, ${ }^{31}$ K. F. Read, ${ }^{128,94}$ K. Redlich ${ }^{84 \pi}$ A. Rehman, ${ }^{22}$ P. Reichelt, ${ }^{69}$ F. Reidt,${ }^{34}$ X. Ren, ${ }^{6}$ R. Renfordt, ${ }^{69}$ A. Reshetin, ${ }^{62}$ J.-P. Revol, ${ }^{10}$ K. Reygers, ${ }^{102}$ V. Riabov, ${ }^{96}$ T. Richert, ${ }^{63,88,80}$ M. Richter, ${ }^{21}$ P. Riedler, ${ }^{34}$ W. Riegler,${ }^{34}$ F. Riggi, ${ }^{28}$ C. Ristea, ${ }^{68}$ S. P. Rode,${ }^{49}$

M. Rodríguez Cahuantzi, ${ }^{44}$ K. Røed, ${ }^{21}$ R. Rogalev, ${ }^{90}$ E. Rogochaya,${ }^{75}$ D. Rohr, ${ }^{34}$ D. Röhrich, ${ }^{22}$ P. S. Rokita, ${ }^{140}$ F. Ronchetti, ${ }^{51}$ E. D. Rosas, ${ }^{70}$ K. Roslon,,${ }^{140}$ P. Rosnet, ${ }^{132}$ A. Rossi,${ }^{56,29}$ A. Rotondi, ${ }^{137}$ F. Roukoutakis, ${ }^{83}$ C. Roy, ${ }^{134}$ P. Roy, ${ }^{107}$ O. V. Rueda, ${ }^{70}$ R. Rui, ${ }^{25}$ B. Rumyantsev, ${ }^{75}$ A. Rustamov, ${ }^{86}$ E. Ryabinkin, ${ }^{87}$ Y. Ryabov, ${ }^{96}$ A. Rybicki, ${ }^{117}$ S. Saarinen, ${ }^{43}$ S. Sadhu, ${ }^{139}$ S. Sadovsky, ${ }^{90}$ K. Šafařík, ${ }^{34}$ S. K. Saha, ${ }^{139}$ B. Sahoo, ${ }^{48}$ P. Sahoo, ${ }^{49}$ R. Sahoo, ${ }^{49}$ S. Sahoo, ${ }^{66}$ P. K. Sahu, ${ }^{66}$ J. Saini, ${ }^{139}$ S. Sakai, ${ }^{131}$ M. A. Saleh, ${ }^{141}$ S. Sambyal, ${ }^{99}$ V. Samsonov, ${ }^{91,96}$ A. Sandoval, ${ }^{72}$ A. Sarkar, ${ }^{73}$ D. Sarkar, ${ }^{139}$ N. Sarkar, ${ }^{139}$ P. Sarma, ${ }^{41}$ M. H. P. Sas, ${ }^{63}$ E. Scapparone, ${ }^{53}$ F. Scarlassara, ${ }^{29}$ B. Schaefer,${ }^{94}$ H. S. Scheid,${ }^{69}$ C. Schiaua, ${ }^{47}$ R. Schicker, ${ }^{102}$ C. Schmidt, ${ }^{104}$ H. R. Schmidt, ${ }^{101}$ M. O. Schmidt, ${ }^{102}$ M. Schmidt, ${ }^{101}$ N. V. Schmidt, ${ }^{69,94}$ J. Schukraft, ${ }^{34}$ Y. Schutz, ${ }^{34,134}$ K. Schwarz, ${ }^{104}$ K. Schweda, ${ }^{104}$ G. Scioli, ${ }^{27}$ E. Scomparin, ${ }^{58}$ M. Šefč́́k, ${ }^{38}$ J. E. Seger, ${ }^{16}$ Y. Sekiguchi, ${ }^{130}$ D. Sekihata, ${ }^{45}$ I. Selyuzhenkov, ${ }^{91,104}$ S. Senyukov, ${ }^{134}$ E. Serradilla, ${ }^{72}$ P. Sett, ${ }^{48}$ A. Sevcenco, ${ }^{68}$ A. Shabanov, ${ }^{62}$ A. Shabetai, ${ }^{113}$ R. Shahoyan, ${ }^{34}$ W. Shaikh, ${ }^{107}$ A. Shangaraev, ${ }^{90}$ A. Sharma ${ }^{98}$ A. Sharma, ${ }^{99}$ M. Sharma ${ }^{99}$ N. Sharma ${ }^{98}$ A. I. Sheikh, ${ }^{139}$ K. Shigaki, ${ }^{45}$ M. Shimomura, ${ }^{82}$ S. Shirinkin,${ }^{64}$ Q. Shou, ${ }^{6,110}$ K. Shtejer, ${ }^{26}$ Y. Sibiriak, ${ }^{87}$ S. Siddhanta, ${ }^{54}$ K. M. Sielewicz ${ }^{34}$ T. Siemiarczuk, ${ }^{84}$ D. Silvermyr, ${ }^{80}$ G. Simatovic, ${ }^{89}$ G. Simonetti, ${ }^{34,103}$ R. Singaraju, ${ }^{139}$ R. Singh, ${ }^{85}$ R. Singh,${ }^{99}$ V. Singhal, ${ }^{139}$ T. Sinha, ${ }^{107}$ B. Sitar, ${ }^{14}$ M. Sitta, ${ }^{32}$ T. B. Skaali, ${ }^{21}$ M. Slupecki, ${ }^{126}$ N. Smirnov, ${ }^{144}$ R. J. M. Snellings, ${ }^{63}$ T. W. Snellman, ${ }^{126}$ J. Sochan, ${ }^{15}$ C. Soncco, ${ }^{109}$ J. Song, ${ }^{18}$ A. Songmoolnak, ${ }^{114}$ F. Soramel,,${ }^{29}$ S. Sorensen, ${ }^{128}$ F. Sozzi, ${ }^{104}$ I. Sputowska, ${ }^{17}$ J. Stachel, ${ }^{102}$ I. Stan ${ }^{68}$ P. Stankus, ${ }^{94}$ E. Stenlund, ${ }^{80}$ D. Stocco, ${ }^{113}$ M. M. Storetvedt, ${ }^{36}$ P. Strmen, ${ }^{14}$ A. A. P. Suaide, ${ }^{120}$ T. Sugitate, ${ }^{45}$ C. Suire, ${ }^{61}$ M. Suleymanov, ${ }^{15}$ M. Suljic, ${ }^{34,25}$ R. Sultanov ${ }^{64}$ M. Šumbera, ${ }^{93}$ S. Sumowidagdo,${ }^{50}$ K. Suzuki, ${ }^{112}$ S. Swain, ${ }^{66}$ A. Szabo, ${ }^{14}$ I. Szarka, ${ }^{14}$ U. Tabassam, ${ }^{15}$ J. Takahashi, ${ }^{121}$ G. J. Tambave ${ }^{22}$ N. Tanaka, ${ }^{131}$ M. Tarhini, ${ }^{113}$ M. G. Tarzila, ${ }^{47}$ A. Tauro, ${ }^{34}$ G. Tejeda Muñoz, ${ }^{44}$ A. Telesca,${ }^{34}$ C. Terrevoli, ${ }^{29}$ B. Teyssier, ${ }^{133}$ D. Thakur, ${ }^{49}$ S. Thakur, ${ }^{139}$ D. Thomas, ${ }^{118}$ F. Thoresen ${ }^{88}$ R. Tieulent, ${ }^{133}$ A. Tikhonov, ${ }^{62}$ A. R. Timmins, ${ }^{125}$ A. Toia, ${ }^{69}$ N. Topilskaya,${ }^{62}$ M. Toppi,${ }^{51}$ F. Torales-Acosta, ${ }^{20}$ S. R. Torres, ${ }^{119}$ S. Tripathy, ${ }^{49}$ S. Trogolo, ${ }^{26}$ G. Trombetta, ${ }^{33}$ L. Tropp,${ }^{38}$ V. Trubnikov, ${ }^{2}$ W. H. Trzaska, ${ }^{126}$ T. P. Trzcinski, ${ }^{140}$ B. A. Trzeciak, ${ }^{63}$ T. Tsuji ${ }^{130}$ A. Tumkin, ${ }^{106}$ R. Turrisi, ${ }^{56}$ T. S. Tveter, ${ }^{21}$ K. Ullaland, ${ }^{22}$ E. N. Umaka, ${ }^{125}$ A. Uras, ${ }^{133}$ G. L. Usai, ${ }^{24}$ A. Utrobicic, ${ }^{97}$ M. Vala, ${ }^{115}$ N. Valle, ${ }^{137}$ J. W. Van Hoorne,${ }^{34}$ M. van Leeuwen,${ }^{63}$ P. Vande Vyvre, ${ }^{34}$ D. Varga,${ }^{143}$ A. Vargas, ${ }^{44}$ M. Vargyas, ${ }^{126}$ R. Varma ${ }^{48}$ M. Vasileiou, ${ }^{83}$ A. Vasiliev,${ }^{87}$ A. Vauthier,${ }^{78}$ O. Vázquez Doce, ${ }^{103,116}$ V. Vechernin, ${ }^{111}$ A. M. Veen, ${ }^{63}$ E. Vercellin, ${ }^{26}$ S. Vergara Limón, ${ }^{44}$ L. Vermunt, ${ }^{63}$ R. Vernet, ${ }^{7}$ R. Vértesi, ${ }^{143}$ L. Vickovic, ${ }^{35}$ J. Viinikainen, ${ }^{126}$ Z. Vilakazi, ${ }^{129}$ O. Villalobos Baillie, ${ }^{108}$ A. Villatoro Tello, ${ }^{44}$ A. Vinogradov, ${ }^{87}$ T. Virgili, ${ }^{30}$ V. Vislavicius, ${ }^{88,80}$ A. Vodopyanov, ${ }^{75}$ M. A. Völkl, ${ }^{101}$ K. Voloshin, ${ }^{64}$ S. A. Voloshin, ${ }^{141}$ G. Volpe,${ }^{33}$ B. von Haller, ${ }^{34}$ I. Vorobyev, ${ }^{116,103}$ D. Voscek, ${ }^{115}$ D. Vranic, ${ }^{104,34}$ J. Vrláková, ${ }^{38}$ B. Wagner, ${ }^{22}$ H. Wang, ${ }^{63}$ M. Wang, ${ }^{6}$ Y. Watanabe, ${ }^{131}$ M. Weber, ${ }^{112}$ S. G. Weber, ${ }^{104}$ A. Wegrzynek, ${ }^{34}$ D. F. Weiser, ${ }^{102}$ S. C. Wenzel, ${ }^{34}$ J. P. Wessels,${ }^{142}$ U. Westerhoff, ${ }^{142}$ A. M. Whitehead, ${ }^{124}$ J. Wiechula, ${ }^{69}$ J. Wikne, ${ }^{21}$ G. Wilk, ${ }^{84}$ J. Wilkinson,${ }^{53}$ G. A. Willems, ${ }^{12,34}$ M. C. S. Williams, ${ }^{53}$ E. Willsher, ${ }^{108}$ B. Windelband, ${ }^{102}$ W. E. Witt, ${ }^{128}$ R. Xu,,${ }^{6}$ S. Yalcin, ${ }^{77}$ K. Yamakawa, ${ }^{45}$ S. Yano, ${ }^{135,45}$ Z. Yin, ${ }^{6}$ H. Yokoyama, ${ }^{131,78}$ I.-K. Yoo, ${ }^{18}$ J. H. Yoon, ${ }^{60}$ V. Yurchenko, ${ }^{2}$ V. Zaccolo, ${ }^{58}$ A. Zaman, ${ }^{15}$ C. Zampolli, ${ }^{34}$ H. J. C. Zanoli, ${ }^{120}$ N. Zardoshti, ${ }^{108}$ A. Zarochentsev, ${ }^{111}$ P. Závada, ${ }^{67}$ N. Zaviyalov, ${ }^{106}$ H. Zbroszczyk, ${ }^{140}$ M. Zhalov, ${ }^{96}$ 
X. Zhang, ${ }^{6}$ Y. Zhang, ${ }^{6}$ Z. Zhang, ${ }^{132,6}$ C. Zhao, ${ }^{21}$ V. Zherebchevskii, ${ }^{111}$ N. Zhigareva, ${ }^{64}$ D. Zhou, ${ }^{6}$ Y. Zhou, ${ }^{88}$ Z. Zhou, ${ }^{22}$ H. Zhu, ${ }^{6}$ J. Zhu, ${ }^{6}$ Y. Zhu, ${ }^{6}$ A. Zichichi, ${ }^{10,27}$ M. B. Zimmermann, ${ }^{34}$ G. Zinovjev, ${ }^{2}$ J. Zmeskal, ${ }^{112}$ and S. Zou ${ }^{6}$

\section{(A Large Ion Collider Experiment Collaboration)}

${ }^{1}$ A.I. Alikhanyan National Science Laboratory (Yerevan Physics Institute) Foundation, Yerevan, Armenia

${ }^{2}$ Bogolyubov Institute for Theoretical Physics, National Academy of Sciences of Ukraine, Kiev, Ukraine

${ }^{3}$ Bose Institute, Department of Physics and Centre for Astroparticle Physics and Space Science (CAPSS),

Kolkata, India

${ }^{4}$ Budker Institute for Nuclear Physics, Novosibirsk, Russia

${ }^{5}$ California Polytechnic State University, San Luis Obispo, California, USA

${ }^{6}$ Central China Normal University, Wuhan, China

${ }^{7}$ Centre de Calcul de l'IN2P3, Villeurbanne, Lyon, France

${ }^{8}$ Centro de Aplicaciones Tecnológicas y Desarrollo Nuclear (CEADEN), Havana, Cuba

${ }^{9}$ Centro de Investigación y de Estudios Avanzados (CINVESTAV), Mexico City and Mérida, Mexico

${ }^{10}$ Centro Fermi-Museo Storico della Fisica e Centro Studi e Ricerche "Enrico Fermi', Rome, Italy

${ }^{11}$ Chicago State University, Chicago, Illinois, USA

${ }^{12}$ China Institute of Atomic Energy, Beijing, China

${ }^{13}$ Chonbuk National University, Jeonju, Republic of Korea

${ }^{14}$ Comenius University Bratislava, Faculty of Mathematics, Physics and Informatics, Bratislava, Slovakia

${ }^{15}$ COMSATS Institute of Information Technology (CIIT), Islamabad, Pakistan

${ }^{16}$ Creighton University, Omaha, Nebraska, USA

${ }^{17}$ Department of Physics, Aligarh Muslim University, Aligarh, India

${ }^{18}$ Department of Physics, Pusan National University, Pusan, Republic of Korea

${ }^{19}$ Department of Physics, Sejong University, Seoul, Republic of Korea

${ }^{20}$ Department of Physics, University of California, Berkeley, California, USA

${ }^{21}$ Department of Physics, University of Oslo, Oslo, Norway

${ }^{22}$ Department of Physics and Technology, University of Bergen, Bergen, Norway

${ }^{23}$ Dipartimento di Fisica dell'Università 'La Sapienza' and Sezione INFN, Rome, Italy

${ }^{24}$ Dipartimento di Fisica dell'Università and Sezione INFN, Cagliari, Italy

${ }^{25}$ Dipartimento di Fisica dell'Università and Sezione INFN, Trieste, Italy

${ }^{26}$ Dipartimento di Fisica dell'Università and Sezione INFN, Turin, Italy

${ }^{27}$ Dipartimento di Fisica e Astronomia dell'Università and Sezione INFN, Bologna, Italy

${ }^{28}$ Dipartimento di Fisica e Astronomia dell'Università and Sezione INFN, Catania, Italy

${ }^{29}$ Dipartimento di Fisica e Astronomia dell'Università and Sezione INFN, Padova, Italy

${ }^{30}$ Dipartimento di Fisica 'E.R. Caianiello' dell'Università and Gruppo Collegato INFN, Salerno, Italy

${ }^{31}$ Dipartimento DISAT del Politecnico and Sezione INFN, Turin, Italy

${ }^{32}$ Dipartimento di Scienze e Innovazione Tecnologica dell'Università del Piemonte Orientale and INFN Sezione di Torino, Alessandria, Italy

${ }^{33}$ Dipartimento Interateneo di Fisica 'M. Merlin' and Sezione INFN, Bari, Italy

${ }^{34}$ European Organization for Nuclear Research (CERN), Geneva, Switzerland

${ }^{35}$ Faculty of Electrical Engineering, Mechanical Engineering and Naval Architecture, University of Split, Split, Croatia

${ }^{36}$ Faculty of Engineering and Science, Western Norway University of Applied Sciences, Bergen, Norway

${ }^{37}$ Faculty of Nuclear Sciences and Physical Engineering, Czech Technical University in Prague, Prague, Czech Republic

${ }^{38}$ Faculty of Science, P.J. Šafárik University, Košice, Slovakia

${ }^{39}$ Frankfurt Institute for Advanced Studies, Johann Wolfgang Goethe-Universität Frankfurt, Frankfurt, Germany

${ }^{40}$ Gangneung-Wonju National University, Gangneung, Republic of Korea

${ }^{41}$ Gauhati University, Department of Physics, Guwahati, India

${ }^{42}$ Helmholtz-Institut für Strahlen- und Kernphysik, Rheinische Friedrich-Wilhelms-Universität Bonn, Bonn, Germany

${ }^{43}$ Helsinki Institute of Physics (HIP), Helsinki, Finland

${ }^{44}$ High Energy Physics Group, Universidad Autónoma de Puebla, Puebla, Mexico

${ }^{45}$ Hiroshima University, Hiroshima, Japan

${ }^{46}$ Hochschule Worms, Zentrum für Technologietransfer und Telekommunikation (ZTT), Worms, Germany

${ }^{47}$ Horia Hulubei National Institute of Physics and Nuclear Engineering, Bucharest, Romania

${ }^{48}$ Indian Institute of Technology Bombay (IIT), Mumbai, India 
${ }^{49}$ Indian Institute of Technology Indore, Indore, India

${ }^{50}$ Indonesian Institute of Sciences, Jakarta, Indonesia

${ }^{51}$ INFN, Laboratori Nazionali di Frascati, Frascati, Italy

${ }^{52}$ INFN, Sezione di Bari, Bari, Italy

${ }^{53}$ INFN, Sezione di Bologna, Bologna, Italy

${ }^{54}$ INFN, Sezione di Cagliari, Cagliari, Italy

${ }^{55}$ INFN, Sezione di Catania, Catania, Italy

${ }^{56}$ INFN, Sezione di Padova, Padova, Italy

${ }^{57}$ INFN, Sezione di Roma, Rome, Italy

${ }^{58}$ INFN, Sezione di Torino, Turin, Italy

${ }^{59}$ INFN, Sezione di Trieste, Trieste, Italy

${ }^{60}$ Inha University, Incheon, Republic of Korea

${ }^{61}$ Institut de Physique Nucléaire d'Orsay (IPNO), Institut National de Physique Nucléaire et de Physique des Particules (IN2P3/CNRS), Université de Paris-Sud, Université Paris-Saclay, Orsay, France

${ }^{62}$ Institute for Nuclear Research, Academy of Sciences, Moscow, Russia

${ }^{63}$ Institute for Subatomic Physics, Utrecht University/Nikhef, Utrecht, Netherlands

${ }^{64}$ Institute for Theoretical and Experimental Physics, Moscow, Russia

${ }^{65}$ Institute of Experimental Physics, Slovak Academy of Sciences, Košice, Slovakia

${ }^{66}$ Institute of Physics, Homi Bhabha National Institute, Bhubaneswar, India

${ }^{67}$ Institute of Physics of the Czech Academy of Sciences, Prague, Czech Republic

${ }^{68}$ Institute of Space Science (ISS), Bucharest, Romania

${ }^{69}$ Institut für Kernphysik, Johann Wolfgang Goethe-Universität Frankfurt, Frankfurt, Germany

${ }^{70}$ Instituto de Ciencias Nucleares, Universidad Nacional Autónoma de México, Mexico City, Mexico

${ }^{71}$ Instituto de Física, Universidade Federal do Rio Grande do Sul (UFRGS), Porto Alegre, Brazil

${ }^{72}$ Instituto de Física, Universidad Nacional Autónoma de México, Mexico City, Mexico

${ }^{73}$ iThemba LABS, National Research Foundation, Somerset West, South Africa

${ }^{74}$ Johann-Wolfgang-Goethe Universität Frankfurt Institut für Informatik,

Fachbereich Informatik und Mathematik, Frankfurt, Germany

${ }^{75}$ Joint Institute for Nuclear Research (JINR), Dubna, Russia

${ }^{76}$ Korea Institute of Science and Technology Information, Daejeon, Republic of Korea

${ }^{77}$ KTO Karatay University, Konya, Turkey

${ }^{78}$ Laboratoire de Physique Subatomique et de Cosmologie, Université Grenoble-Alpes, CNRS-IN2P3, Grenoble, France

${ }^{79}$ Lawrence Berkeley National Laboratory, Berkeley, California, USA

${ }^{80}$ Lund University Department of Physics, Division of Particle Physics, Lund, Sweden

${ }^{81}$ Nagasaki Institute of Applied Science, Nagasaki, Japan

${ }^{82}$ Nara Women's University (NWU), Nara, Japan

${ }^{83}$ National and Kapodistrian University of Athens, School of Science, Department of Physics, Athens, Greece

${ }^{84}$ National Centre for Nuclear Research, Warsaw, Poland

${ }^{85}$ National Institute of Science Education and Research, Homi Bhabha National Institute, Jatni, India

${ }^{86}$ National Nuclear Research Center, Baku, Azerbaijan

${ }^{87}$ National Research Centre Kurchatov Institute, Moscow, Russia

${ }^{88} \mathrm{Niels}$ Bohr Institute, University of Copenhagen, Copenhagen, Denmark

${ }^{89}$ Nikhef, National institute for subatomic physics, Amsterdam, Netherlands

${ }^{90}$ NRC Kurchatov Institute IHEP, Protvino, Russia

${ }^{91}$ NRNU Moscow Engineering Physics Institute, Moscow, Russia

${ }^{92}$ Nuclear Physics Group, STFC Daresbury Laboratory, Daresbury, United Kingdom

${ }^{93}$ Nuclear Physics Institute of the Czech Academy of Sciences, $\check{R} e z ̌$ u Prahy, Czech Republic

${ }^{94}$ Oak Ridge National Laboratory, Oak Ridge, Tennessee, USA

${ }^{95}$ Ohio State University, Columbus, Ohio, USA

${ }^{96}$ Petersburg Nuclear Physics Institute, Gatchina, Russia

${ }^{97}$ Physics department, Faculty of science, University of Zagreb, Zagreb, Croatia

${ }^{98}$ Physics Department, Panjab University, Chandigarh, India

${ }^{99}$ Physics Department, University of Jammu, Jammu, India

${ }^{100}$ Physics Department, University of Rajasthan, Jaipur, India

${ }^{101}$ Physikalisches Institut, Eberhard-Karls-Universität Tübingen, Tübingen, Germany

${ }^{102}$ Physikalisches Institut, Ruprecht-Karls-Universität Heidelberg, Heidelberg, Germany

${ }^{103}$ Physik Department, Technische Universität München, Munich, Germany 


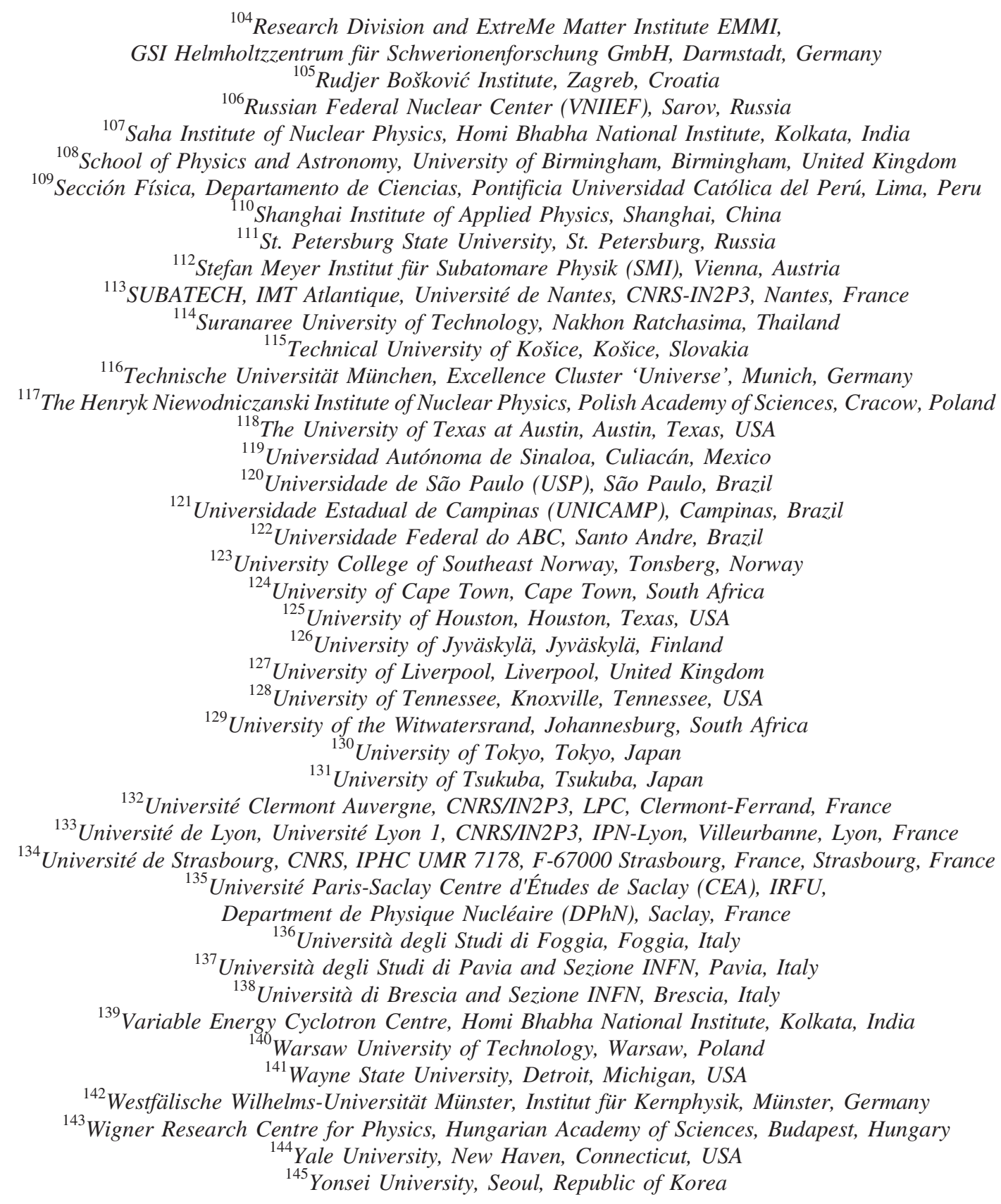

${ }^{\dagger}$ Deceased.

*Also at: Dipartimento DET del Politecnico di Torino, Turin, Italy.

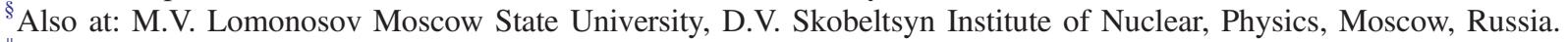

"Also at: Department of Applied Physics, Aligarh Muslim University, Aligarh, India.

"Also at: Institute of Theoretical Physics, University of Wroclaw, Poland. 